University of Wollongong

Research Online

Faculty of Engineering and Information

Faculty of Engineering and Information

Sciences - Papers: Part A

Sciences

$1-1-2015$

\title{
A subspace projection approach for wall clutter mitigation in through-the- wall radar imaging
}

Fok Hing Chi Tivive

University of Wollongong, tivive@uow.edu.au

Abdesselam Bouzerdoum

University of Wollongong, bouzer@uow.edu.au

Moeness Amin

Villanova University, mamin@uow.edu.au

Follow this and additional works at: https://ro.uow.edu.au/eispapers

Part of the Engineering Commons, and the Science and Technology Studies Commons

Research Online is the open access institutional repository for the University of Wollongong. For further information contact the UOW Library: research-pubs@uow.edu.au 


\title{
A subspace projection approach for wall clutter mitigation in through-the-wall radar imaging
}

\begin{abstract}
One of the main challenges in through-the-wall radar imaging (TWRI) is the strong exterior wall returns, which tend to obscure indoor stationary targets, rendering target detection and classification difficult, if not impossible. In this paper, an effective wall clutter mitigation approach is proposed for TWRI that does not require knowledge of the background scene nor does it rely on accurate modeling and estimation of wall parameters. The proposed approach is based on the relative strength of the exterior wall returns compared to behind-wall targets. It applies singular value decomposition to the data matrix constructed from the space-frequency measurements to identify the wall subspace. Orthogonal subspace projection is performed to remove the wall electromagnetic signature from the radar signals. Furthermore, this paper provides an analysis of the wall and target subspace characteristics, demonstrating that both wall and target subspaces can be multidimensional. While the wall subspace depends on the wall type and building material, the target subspace depends on the location of the target, the number of targets in the scene, and the size of the target. Experimental results using simulated and real data demonstrate the effectiveness of the subspace projection method in mitigating wall clutter while preserving the target image. It is shown that the performance of the proposed approach, in terms of the improvement factor of the target-to-clutter ratio, is better than existing approaches and is comparable to that of background subtraction, which requires knowledge of a reference background scene.
\end{abstract}

\section{Keywords}

projection, approach, wall, clutter, subspace, mitigation, radar, imaging

Disciplines

Engineering | Science and Technology Studies

\section{Publication Details}

F. Tivive, A. Bouzerdoum \& M. G. Amin, "A subspace projection approach for wall clutter mitigation in through-the-wall radar imaging," IEEE Transactions on Geoscience and Remote Sensing, vol. 53, (4) pp. 2108-2122, 2015. 


\title{
A Subspace Projection Approach for Wall Clutter Mitigation in Through-the-Wall Radar Imaging
}

\author{
Fok Hing Chi Tivive, Member, IEEE, Abdesselam Bouzerdoum, Senior Member, IEEE, \\ and Moeness G. Amin, Fellow, IEEE
}

\begin{abstract}
One of the main challenges in through-the-wall radar imaging (TWRI) is the strong exterior wall returns, which tend to obscure indoor stationary targets, rendering target detection and classification difficult, if not impossible. In this paper, an effective wall clutter mitigation approach is proposed for TWRI that does not require knowledge of the background scene nor does it rely on accurate modeling and estimation of wall parameters. The proposed approach is based on the relative strength of the exterior wall returns compared to behind-wall targets. It applies singular value decomposition to the data matrix constructed from the space-frequency measurements to identify the wall subspace. Orthogonal subspace projection is performed to remove the wall electromagnetic signature from the radar signals. Furthermore, the paper provides an analysis of the wall and target subspace characteristics, demonstrating that both the wall and target subspaces can be multi-dimensional. While the wall subspace depends on the wall-type and building material, the target subspace depends on the location of the target, the number of targets in the scene, and the size of the target. Experimental results using simulated and real data demonstrate the effectiveness of the subspace projection method in mitigating wall clutter while preserving the target image. It is shown that the performance of the proposed approach, in terms of the improvement factor of the target-to-clutter ratio, is better than existing approaches and is comparable to that of background subtraction, which requires knowledge of a reference background scene.
\end{abstract}

Index Terms-Through-the-wall radar imaging, wall clutter removal, singular value decomposition, wall subspace, target subspace, subspace projection.

\section{INTRODUCTION}

Through-the-Wall Radar Imaging (TWRI) is an emerging technology of increasing interest. The main objective is to sense through the wall and inside enclosed building structures by using electromagnetic (EM) waves for determining the building layouts, discerning the intent of activities inside the building, and detecting, identifying and tracking moving targets. This type of technology is highly desirable in search-andrescue missions, behind-wall target detection, and surveillance and reconnaissance in urban environments [1]-[5]. One of the main issues of imaging stationary targets inside a building is

F. H. C. Tivive and A. Bouzerdoum are with the School of Electrical, Computer and Telecommunications Engineering, University of Wollongong, Northfields Avenue, Wollongong NSW 2522, Australia (Email: \{tivive, bouzer\}@uow.edu.au). This work is supported by a grant from the Australian Research Council (ARC).

M. G. Amin is with the Radar Imaging Lab, Center for Advanced Communications, Villanova University, PA 19085 USA (Email: moeness.amin@villanova.edu). This work of Dr. Amin is supported by Office of Naval Research under Grant N00014-11-0576. the strong clutter induced by the exterior wall, which is usually a highly reflective and attenuative medium.

Most TWRI studies dealing with stationary targets [6][8] assume to have access of a background or reference scene, where background subtraction is performed on the raw data prior to applying an image formation method for scene reconstruction. This approach, though effective in removing wall returns, is not feasible in practice. Therefore, different approaches have been proposed to deal with strong wall reflections without relying on the background scene data [9][14]. From the received signals, especially the first wave arrivals, it is possible to estimate the front wall parameters, such as dielectric constant and thickness [11]. The estimated parameters can be used to model the electromagnetic wall returns, which are subsequently subtracted from the total radar returns, rendering the received signals free of wall reflections. This approach requires accuracy in parameter estimation and modeling. Another method of suppressing the wall reflections is to use three antenna arrays placed parallel to the wall at different heights, where the upper and lower arrays comprise receivers and the middle array consists of transmitters [10]. A simple subtraction of the radar returns from the lower and upper arrays can lead to wall clutter reduction. Due to the receiver symmetry with respect to the transmitter, the contribution of the reflection from the wall in the difference signal is suppressed. In this scheme, two additional arrays are required and the effect of the subtraction operation on the target reflections is unknown and cannot be controlled. A spatial filtering method was proposed for wall clutter mitigation [9]. This method relies on invariance of the wall characteristic and is based on the assumption that the wall returns have the same characteristics with changing antenna location. This spatial invariance can be horizontal, vertical, or along both dimensions in the wall plane. Thus, a notch filter was applied across the antenna array to remove the zerofrequency or low spatial frequencies, which capture constant or slowly varying wall returns. It is noted, however, that the filtering method is effective only for homogeneous or nearhomogeneous walls and at low operating frequencies.

In this paper, we assume that the scene is stationary, and hence change detection or Doppler/microDoppler processing is not applicable for wall clutter removal, and deletion of animate and inanimate targets [16]-[18]. We present a new subspace method for mitigating wall clutter, or at least significantly suppressing it, to reveal the targets behind the wall. The proposed technique first identifies the wall clutter and target signal subspaces using singular value decomposition (SVD); 
then, it projects the radar signal onto a subspace orthogonal to the wall subspace. SVD has been used previously in groundpenetrating radar (GPR) to improve the signal-to-noise ratio (SNR) of the radar images [19], [20]; the B-scan image is decomposed into several eigen-images and the first eigenimage is considered the target image. SVD has been used in TWRI to remove the wall clutter and to detect behindthe-wall targets from B-scan images [12]-[15]. The wall clutter and the target reflections are assumed to reside in the first and second eigen-images, respectively, whereas the remaining eigen-images contain noise. Another SVD-based method was proposed to remove wall clutter in the formed image, where the wall reflections still reside in the first eigenimages and the target reflections span several eigen-images [15]. However, recently, we have shown that the wall clutter is generally characterized by a high dimensional subspace [21], [22]. Furthermore, the weak wall singular components may interleave with the target singular components. Therefore, a more effective technique is required to separate the wall and target subspaces since the first singular component is unlikely to account for all wall returns.

This paper extends our previous work [21], [22] in both analysis and experimentation. It considers SVD of the data matrix constructed from stepped-frequency matched filtered measurements obtained at different antenna positions. In so doing, it operates on the data and not on the beamformed image. The results of the two operations are entirely different due to the target localization through coherent combining. We show that, in near field imaging, the wall returns can span a multidimensional subspace, which depends, among other factors, on the periodic structure of the wall, the frequency response, the uniformity of the wall thickness, and the array geometry. Moreover, the target reflections can span a subspace whose dimension depends on the target size, the target location, the number of targets, and the configuration of the antenna array. Both empirical data and simulations confirm that the wall returns generally span a multidimensional subspace, where the significant target singular components can interleave with some of the weak wall singular components. The paper conducts a comprehensive analysis of the wall and target eigen-subspaces. Furthermore, it presents a subspace classification method to segregate between the target and wall subspaces. A subspace projection method is then proposed for wall clutter mitigation, which works on the space-frequency data matrix instead of the formed image.

The remainder of the paper is organized as follows. The next section presents the geometric model of TWRI and describes delay-and-sum (DS) beamforming for image formation. Section III presents the analysis of the wall and target eigen-subspaces supported by simulation results. Section IV describes the proposed subspace projection approach for wall clutter mitigation. Experimental results using real data are given in Section V. Finally, the conclusion is presented in Section VI.

\section{Through Wall Radar Imaging Signal Model}

This section presents the TWRI signal model used to explain the proposed wall clutter mitigation approach. The imaging scheme is derived for free-space and then extended to imaging behind a homogeneous wall. The geometric model of TWRI as described in [5] is used to estimate the signal propagation delay in the presence of a homogeneous wall.

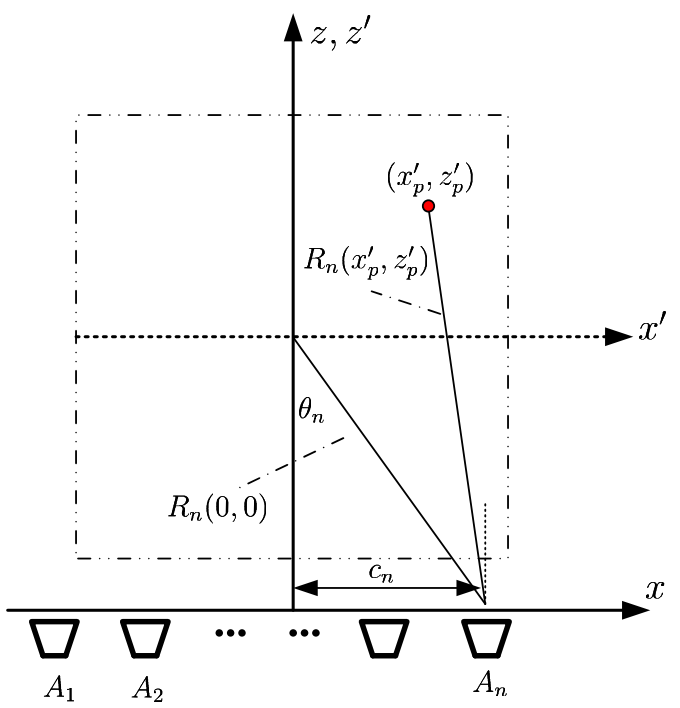

(a)

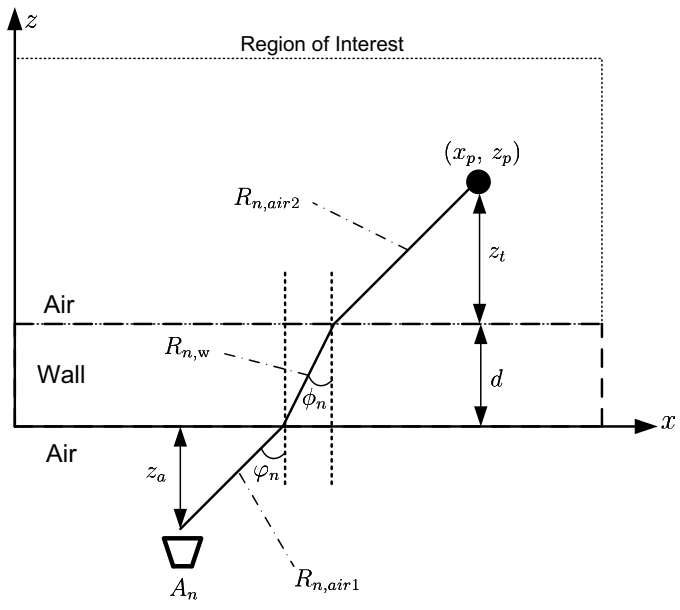

(b)

Fig. 1. Through-the-wall radar imaging geometry: (a) in free-space and (b) through the wall.

In free-space, the geometric model of a TWRI system is depicted in Fig. 1(a). Here, a ground-based mono-static SAR system is used to synthesize an $N$-element linear array. A local coordinate system is defined to represent the region of interest with the horizontal and vertical axes denoted as $x^{\prime}$ and $z^{\prime}$, respectively. The center of the scene is at $(0,0)$ and $\theta_{n}$ is the viewing angle of the $n$-th antenna. Let $R_{n}(0,0)$ and $c_{n}$ denote the distance of the $n$-th antenna to the center of the scene and to the center of the array aperture, respectively. The distance from the $n$-th antenna to the pixel location $\left(x_{p}^{\prime}, z_{p}^{\prime}\right)$ within the region of interest is denoted by $R_{n}\left(x_{p}^{\prime}, z_{p}^{\prime}\right)$ and can be computed as

$$
\begin{aligned}
R_{n}\left(x_{p}^{\prime}, z_{p}^{\prime}\right)= & \left(R_{n}(0,0)^{2}+2 R_{n}(0,0) z_{p}^{\prime} \cos \left(\theta_{n}\right)\right. \\
& \left.-2 R_{n}(0,0) x_{p}^{\prime} \sin \left(\theta_{n}\right)+x_{p}^{\prime 2}+z_{p}^{\prime 2}\right)^{\frac{1}{2}}
\end{aligned}
$$


The viewing angle $\theta_{n}$ of the $n$-th antenna is given by

$$
\theta_{n}=\sin ^{-1}\left(\frac{c_{n}}{R_{n}(0,0)}\right) \text {. }
$$

Without loss of generality, let us assume a single target located at $\left(x_{p}^{\prime}, z_{p}^{\prime}\right)$. The two-way propagation delay $\tau_{n}\left(x_{p}^{\prime}, z_{p}^{\prime}\right)$ from the $n$-th antenna to the target is given by

$$
\tau_{n}\left(x_{p}^{\prime}, z_{p}^{\prime}\right)=\frac{2 R_{n}\left(x_{p}^{\prime}, z_{p}^{\prime}\right)}{c},
$$

where $c$ is the speed of light in free space.

When there is a homogeneous wall in front of the radar system as shown in Fig. 1(b), the two-way propagation delay of the radar signal from the $n$-th antenna to the target is given by

$$
\begin{aligned}
\tau_{n}\left(x_{p}, z_{p}\right)=\frac{2}{c}\left(R_{n, \text { air } 1}\left(x_{p}, z_{p}\right)+\right. & \sqrt{\epsilon} R_{n, \mathrm{w}}\left(x_{p}, z_{p}\right) \\
& \left.+R_{n, \operatorname{air} 2}\left(x_{p}, z_{p}\right)\right),
\end{aligned}
$$

where $\epsilon$ is the relative permittivity of the wall and $R_{n, \text { air } 1}\left(x_{p}, z_{p}\right), R_{n, \mathrm{w}}\left(x_{p}, z_{p}\right)$, and $R_{n, \text { air } 2}\left(x_{p}, z_{p}\right)$ denote, respectively, the distances traveled by the signal from the $n$-th antenna to the target at location $\left(x_{p}, z_{p}\right)$ before, through, and after the wall. These distances can be estimated as follows [8]:

$$
\begin{aligned}
R_{n, \text { air } 1}\left(x_{p}, z_{p}\right) & =\frac{z_{a}}{\cos \left(\varphi_{n}\left(x_{p}, z_{p}\right)\right)}, \\
R_{n, \mathrm{w}}\left(x_{p}, z_{p}\right) & =\frac{d}{\cos \left(\phi_{n}\left(x_{p}, z_{p}\right)\right)}, \\
R_{n, \text { air } 2}\left(x_{p}, z_{p}\right) & =\frac{z_{t}}{\cos \left(\varphi_{n}\left(x_{p}, z_{p}\right)\right)},
\end{aligned}
$$

where $z_{a}$ is the standoff distance from the antenna array to the wall, $d$ is the wall thickness, $z_{t}$ is the distance from the wall to the target, and $\varphi_{n}\left(x_{p}, z_{p}\right)$ and $\phi_{n}\left(x_{p}, z_{p}\right)$ are the angles of incidence and refraction from the $n$-th antenna to the target at location $\left(x_{p}, z_{p}\right)$, respectively. To image the behind-the-wall scene, a stepped-frequency signal is synthesized by emitting monochromatic signals with frequencies equispaced over the desired bandwidth $\omega_{M-1}-\omega_{0}$ :

$$
\omega_{m}=\omega_{0}+m \Delta \omega, \quad \text { for } m=0, \ldots, M-1,
$$

where $\omega_{0}$ is the lowest frequency in the desired frequency band, $\Delta \omega$ is the frequency step size, and $M$ is the total number of frequencies.

There are several approaches for image formation including tomographic approaches [23], differential synthetic aperture radar (SAR) [24], compressed sensing [25], and adaptive beamformers [26], [27]. Here, we employ delay-and-sum beamforming to compute the complex amplitude of the pixel, which is given by

$$
I(x, z)=\frac{1}{N M} \sum_{n=0}^{N-1} \sum_{m=0}^{M-1} s(m, n) \exp \left(j \omega_{m} \tau_{n}(x, z)\right),
$$

where $s(m, n)$ is the radar signal at the $m$-th frequency received by the $n$-th antenna and $\tau_{n}(x, z)$ is the focusing delay for the pixel at location $(x, z)$ with respect to the $n$-th antenna, including the propagation through the wall. Before describing the proposed wall clutter mitigation method, we first present the analysis of the wall and target eigen-subspaces.

\section{Analysis of Wall And TARget EIGEN-Subspaces}

Several SVD-based wall clutter mitigation approaches have been proposed which assume that the wall reflections are characterized by the first singular vector associated with the most dominant singular value [12]-[15]. In [21] and [22], we have shown that multiple singular vectors can span the wall subspace. In this section, we investigate the factors affecting the wall and target eigen-subspaces. The dimension of the wall subspace is related to, among other factors, the wall heterogeneity, the wall thickness uniformity, and the antenna array configuration. For the target subspace, its dimension is affected by the target location, the target size, the number of targets behind the wall, and the configuration of the array aperture. Numerical simulations using XFDTD are included to support the analysis of the wall and target subspaces.

\section{A. Eigen-Structure of Wall Subspace}

In practical TWRI applications, we often deal with two types of walls: homogeneous and heterogeneous walls. The following two subsections analyze the eigen-structure of the wall subspace, using both types of walls.

1) Homogeneous Wall: A homogeneous wall can be modeled as a uniform dielectric slab of thickness $d$ and dielectric constant $\epsilon$. Assuming that the signal is transmitted perpendicularly to the surface of the wall, the wall return of a homogeneous wall is calculated based on the plane wave Fresnel reflection and transmission coefficients obtained from Maxwells equations [28]. Let $\rho$ be the local Fresnel reflection coefficient, given by

$$
\rho=\frac{1-\sqrt{\epsilon}}{1+\sqrt{\epsilon}} .
$$

The reflection coefficient at the $m$-th frequency $\Gamma_{m}$ can be written as

$$
\Gamma_{m}=\frac{\rho\left(1-\exp \left(-2 j \sqrt{\epsilon} k_{m} d\right)\right)}{1-\rho^{2} \exp \left(-2 j \sqrt{\epsilon} k_{m} d\right)},
$$

where $k_{m}=\omega_{m} / c$ is the wave number. The radar signal backscattered from the wall and received by the $n$-th antenna can be expressed as

$$
s_{\mathrm{w}}(m, n)=\frac{G_{m} \lambda_{m}}{8 \pi} \frac{\exp \left(-j 2 k_{m} z_{n}\right)}{z_{n}} \Gamma_{m},
$$

where $z_{n}$ is the distance between the $n$-th antenna and the wall, $\lambda_{m}$ is the wavelength of the $m$-th monochromatic signal, and $G_{m}$ is the antenna gain at the $m$-th frequency [11]. The wall backscattered signals received by the $N$-element array can be arranged into a matrix $\Phi_{\mathrm{w}} \in \mathcal{C}^{M \times N}$, where each column contains the signal received at one antenna location and each row contains the signals from one frequency,

$$
\Phi_{\mathrm{w}}=\left[\phi_{m n}\right],
$$

where $\phi_{m n}=s_{\mathrm{w}}(m, n)$. Assuming that the antenna gain and wall reflection coefficient do not change with antenna location, $\Phi_{\mathrm{w}}$ can be expressed as the product of a diagonal matrix $A$, containing the antenna gains and reflection coefficients (as 
a function of frequency), with an $M \times N$ matrix $B$ which depends on the antenna standoff distance:

$$
\Phi_{\mathrm{w}}=A B
$$

where

$$
A=\operatorname{diag}\left(G_{0} \lambda_{0} \Gamma_{0}, \ldots, G_{M-1} \lambda_{M-1} \Gamma_{M-1}\right)
$$

and

$$
B=\left[b_{m n}\right]
$$

with

$$
b_{m n}=\frac{\exp \left(-j 2 k_{m} z_{n}\right)}{8 \pi z_{n}} .
$$

The wall eigen-subspace can be obtained by applying SVD and identifying the singular vectors containing the wall returns. Since $A$ is a full rank diagonal matrix, it follows that $\operatorname{rank}\left(\Phi_{\mathrm{w}}\right)=\operatorname{rank}(B)$; therefore, the wall subspace dimension is determined by the rank of $B$. Furthermore, it is clear from (17) that the columns of $B$ depend on the antenna standoff distance $z_{n}$; the rows of $B$ depend on the frequency. In practice, the number of antennas $N$ is smaller than the number of frequencies $M$. Thus, the rank of $B$ is determined by the standoff distance of the antenna to the wall. Although, the gain of the transceiver in a synthesized array aperture varies with frequencies and not antenna locations, it has no effect on the rank of the matrix $\Phi_{\mathrm{w}}$, and therefore does not change the dimension of the wall subspace.

There are two cases where the signals backscattered from a homogeneous wall span a multidimensional subspace: the antenna array is not perfectly aligned (parallel to) the wall surface or the wall exhibits nonuniform thickness along the antenna array. In the first case, each antenna is positioned at a different standoff distance $z_{n}$ from the wall; thus, the columns of $B$ become linearly independent, thereby increasing the rank of the matrix and the dimension of the wall subspace. In the second case, due to the variations in the wall thickness, the two-way propagation delay of the signal reflected from the back of the wall varies from one antenna to another, causing the signals received across the antennas to be different from each other, and hence increasing the dimension of the wall subspace. These two cases are investigated using numerical simulations with XFDTD software. The first numerical simulation scenario consists of a homogeneous wall of thickness $0.15 \mathrm{~m}$ and a dielectric constant 7.6 placed in front of the radar at a standoff distance of $1 \mathrm{~m}$. A 51-element antenna array of size $1.2 \mathrm{~m}$ is synthesized for imaging. The excitation signal is a modulated Gaussian pulse which covers the frequency range from 2 to $3 \mathrm{GHz}$. The time domain responses are transformed into the frequency domain and sampled to produce the stepped-frequency signals which are arranged into a matrix $\Phi_{\mathrm{w}}$. Using SVD, the matrix $\Phi_{\mathrm{w}}$ can be decomposed as

$$
\Phi_{\mathrm{w}}=U \Sigma V^{H}
$$

where ${ }^{H}$ denotes Hermitian transpose, $U=\left[\boldsymbol{u}_{1}, \ldots, \boldsymbol{u}_{M}\right]$ and $V=\left[\boldsymbol{v}_{1}, \ldots, \boldsymbol{v}_{N}\right]$ are unitary matrices containing the left and right singular vectors, respectively, and $\Sigma$ is a rectangular matrix of the same size as $\Phi_{\mathrm{w}}$ with singular values $\sigma_{i}$ on the main diagonal arranged in decreasing order, i.e., $\sigma_{1} \geq \sigma_{2} \geq \ldots \geq \sigma_{N} \geq 0$. Ideally, a homogeneous wall subspace is spanned by the first singular vector associated with the dominant singular value. Perturbations in the remaining singular values are considered as subspace distortions. We define the subspace distortion index of a homogeneous wall $\delta_{s}$ as the fraction of power carried by the non-dominant singular components,

$$
\delta_{s}=\frac{\sum_{i=2}^{N} \sigma_{i}^{2}}{\sum_{i=1}^{N} \sigma_{i}^{2}} .
$$

In the first case, the antenna array is tilted at an angle with respect to the wall surface. The subspace distortion index of a homogeneous wall is computed while varying the tilt angle from 1 to 10 degree. Figure 2(a) illustrates the variations of the subspace distortion index as a function of the tilt angle, and Fig. 2(b) depicts the normalized singular values of the wall subspace at a subspace distortion index of 0.15 . A misalignment of five degree with respect to the surface of the wall produces a subspace distortion index of 0.15 , resulting in 16 nonzero singular values, as shown in Fig. 2(b). Therefore, we can conclude that the signals backscattered from a homogeneous wall that is not parallel to the antenna array span a multi-dimensional subspace.

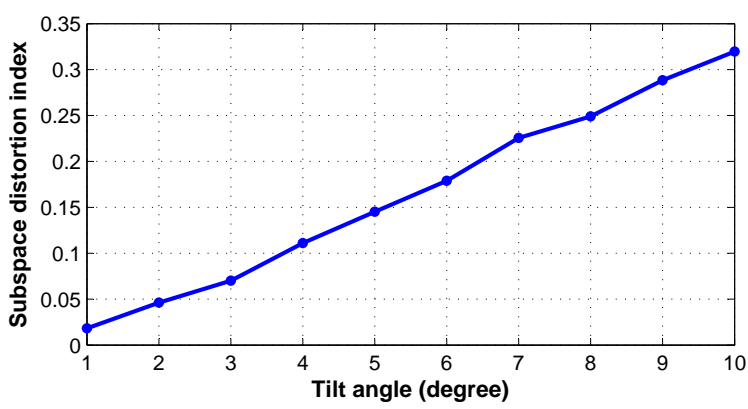

(a)

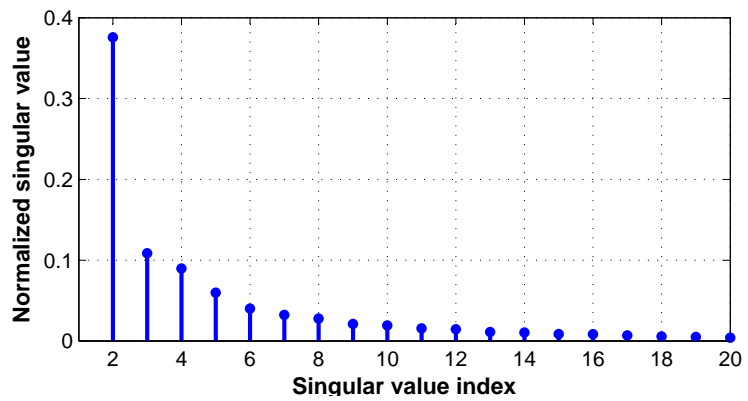

(b)

Fig. 2. Perturbation analysis in the antenna standoff distance: (a) subspace distortion index as a function of the tilt angle and (b) the first 20 normalized singular values at a tilt angle of five degree. For clarity, the first singular value is omitted; the other singular values are normalized with the first one.

In the second case, the wall thickness is increased gradually along the antenna array: the wall thickness in front of the first antenna is $d$ and at the last antenna is $d+\Delta d$. In the simulations, the parameter $\Delta d$ is increased from 0.03 to $0.3 \mathrm{~m}$ while $d$ is fixed at $0.15 \mathrm{~m}$. The subspace distortion index computed as a function of the relative variations in the wall thickness is shown in Fig. 3(a). Figure 3(b) illustrates the normalized wall singular values at a relative variation of 
$0.06(\Delta d / d=0.06)$ in the wall thickness. The numerical simulations show that when the homogeneous wall does not have uniform thickness, the wall reflections span a multidimensional subspace.

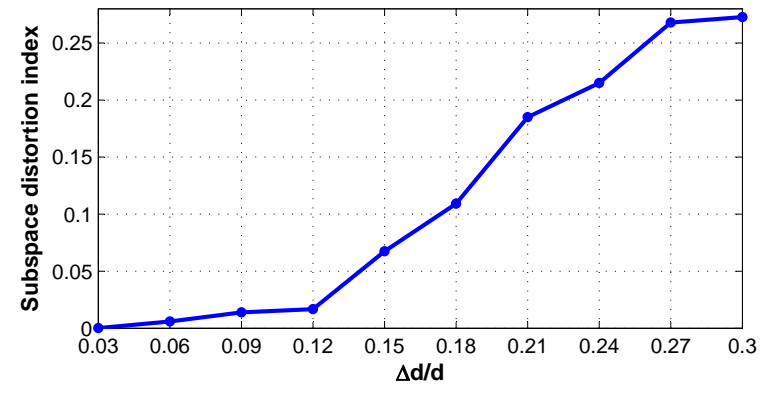

(a)

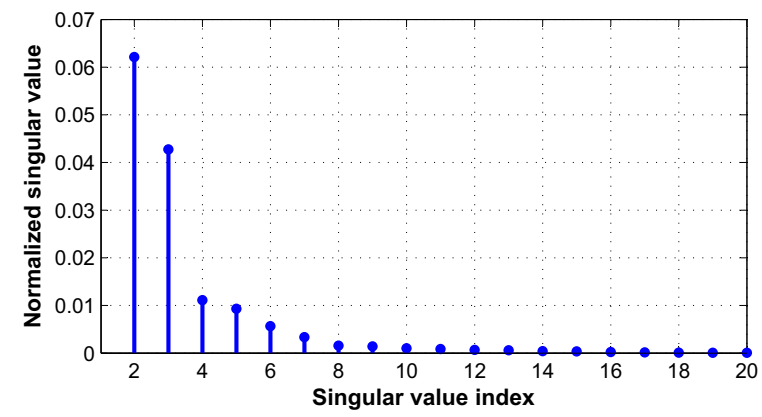

(b)

Fig. 3. Perturbation analysis in the wall thickness: (a) subspace distortion index as a function of the relative variations in the wall thickness and (b) the first 20 normalized singular values excluding the first one at a relative perturbation of 0.06 .

\section{B. Heterogeneous Wall}

A wall is considered heterogeneous when the dielectric properties of its building material vary along either or both dimensions, i.e., height and width. If a wall is built from several vertical planes whose dielectric constants vary along the horizontal antenna array, it is clear from (11) that the reflection coefficient will be a function of the antenna location; thereby, the columns of the matrix $\Phi_{\mathrm{w}}$ become linearly independent and the wall subspace increases. In practice, most heterogeneous walls are built in such a way that they exhibit some periodicity. For example, walls constructed from cinderblock or crossbar reinforced concrete are two-dimensional periodic heterogeneous walls, whereas drywalls with vertical wooden studs and reinforced concrete walls with vertical rebars only are one-dimensional periodic walls. Due to the fast-fading phenomenon caused by the wall heterogeneity, this type of walls is analyzed by either using electromagnetic simulation tool or performing real experiments.

For eigen-subspace analysis, we conduct several numerical simulations using two kinds of heterogeneous walls: hollow concrete block wall and reinforced concrete wall. The hollow concrete block wall is built using cinderblocks of size $0.2 \mathrm{~m}$ $\times 0.4 \mathrm{~m}$ with a thickness of $0.15 \mathrm{~m}$. The reinforced concrete wall has a thickness of $0.15 \mathrm{~m}$ and consists of $0.025 \mathrm{~m}$ thick vertical metallic rebars spacing at an interval of $0.2 \mathrm{~m}$. The
TWRI scene devoid of targets is illuminated with a modulated Gaussian pulse centered at $1.5 \mathrm{GHz}$. The time domain responses obtained from each of these walls are transformed into stepped-frequency signals covering the frequency band of 2 to $3 \mathrm{GHz}$; they are then arranged into a matrix $\Phi_{\mathrm{w}}$. Figure 4 shows the DS-beamformed images and the normalized singular values of the signal matrix for both types of heterogeneous walls: Figures 4(a) and (b) are for the hollow concrete block wall, and Figs. 4(c) and (d) are for the reinforced concrete wall. The number of nonzero singular values in Figs. 4(b) and (d) indicates that the rank of the matrix $\Phi_{\mathrm{w}}$ is greater than one. Thus, we conclude that the wall returns from a heterogeneous wall span a multi-dimensional subspace.

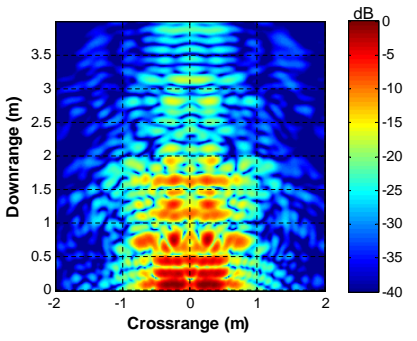

(a)

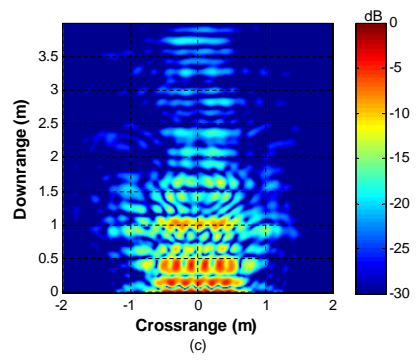

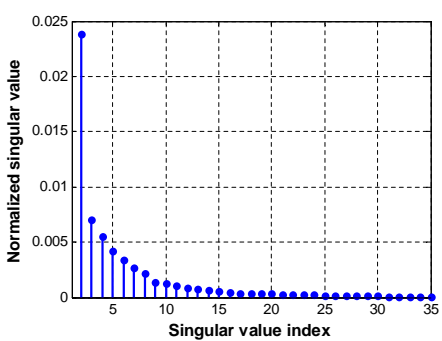

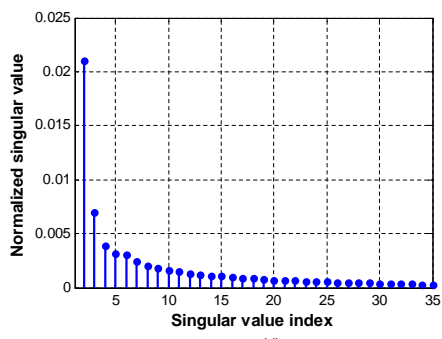

(d)
Fig. 4. Eigen-structure of a heterogeneous wall: (a) image of the hollow concrete block wall, (b) its normalized singular values, (c) image of the reinforced concrete wall with vertical rebars only, and (d) its normalized singular values. For clarity, the first singular value is omitted; the other singular values are normalized with the first one.

To summarize, the wall subspace is not necessarily characterized by a single singular vector, but can be spanned by multiple singular vectors. There are several factors that affect the dimension of the wall subspace, namely, the wall electromagnetic characteristics, the wall thickness uniformity, and the configuration of the antenna array. The next subsection presents an analysis of the target subspace.

\section{Eigen-Structure of Target Subspace}

In this section, we analyze the eigen-subspace of a target, where the received signals comprise only the target returns. First, we consider a point target with frequency-dependent reflection coefficient $\sigma_{m}$ located at the location $\left(x_{p}^{\prime}, z_{p}^{\prime}\right)$. The target signal received by the $n$-th antenna can be written as

$$
s_{\mathrm{t}}(m, n)=\frac{\sigma_{m} G_{m} \lambda_{m}}{4 \pi} \exp \left(-j \omega_{m} \tau_{n}\left(x_{p}^{\prime}, z_{p}^{\prime}\right)\right),
$$

The target signals received across the antenna array are arranged into the matrix $\Phi_{t}$ :

$$
\Phi_{\mathrm{t}}=A B
$$


where

$$
A=\operatorname{diag}\left(\frac{\sigma_{0} G_{0} \lambda_{0}}{4 \pi}, \frac{\sigma_{1} G_{1} \lambda_{1}}{4 \pi}, \ldots, \frac{\sigma_{M-1} G_{M-1} \lambda_{M-1}}{4 \pi}\right)
$$

and

$$
B=\left[\exp \left(-j \omega_{m} \tau_{n}\left(x_{p}^{\prime}, z_{p}^{\prime}\right)\right)\right]
$$

Since the two-way propagation delay between the $n$-th antenna and the target $\tau_{n}\left(x_{p}^{\prime}, z_{p}^{\prime}\right)$ is dependent on the antenna location, it is expected to vary from one antenna location to another, causing the columns of the matrix $B$ in (23) to become linearly independent. Therefore, the rank of $\Phi_{\mathrm{t}}$ changes with the target location. For example, a target placed at the center of the array will reduce the rank of $\Phi_{t}$ since the signal propagation delays from the antennas on the left half of the array are the same as those on the right half of the array, i.e., $\tau_{0}\left(x_{p}^{\prime}, z_{p}^{\prime}\right)=\tau_{N-1}\left(x_{p}^{\prime}, z_{p}^{\prime}\right), \tau_{1}\left(x_{p}^{\prime}, z_{p}^{\prime}\right)=\tau_{N-2}\left(x_{p}^{\prime}, z_{p}^{\prime}\right)$, etc. In a recent study [21], we have shown that reflections from a point target span a multi-dimensional subspace, which depends on the number of targets in the scene and the configuration of the antenna array. The target location with respect to the radar also influences the target subspace dimension.

Next, we investigate the dimension of the target subspace under two imaging scenarios: short-range and long-range, where the target is placed close to or far from the radar system. In the former, the viewing angle $\theta_{n}$ of the antenna varies considerably across the array aperture, causing the distance traveled by the signal from each antenna to the target to be different. Based on the propagation delay given in (3), it is clear that the target signal related to the viewing angle, and therefore, the number of linearly independent columns in the matrix $\Phi_{\mathrm{t}}$ increases when the viewing angle varies markedly across the array aperture. On the other hand, for a long-range target, the changes in the viewing angle across the antenna array are much smaller, resulting in almost the same distance between each antenna element and the target; therefore, the target subspace is narrower compared to that of a short-range target. To illustrate this, a target is placed at two different locations: a short-range at $(0,1.2) \mathrm{m}$ and a long-range at $(0,6.2) \mathrm{m}$. The formed images and the singular values for both cases are shown in Fig. 5. Figures 5(a) and (b) depict, respectively, the formed image and the singular values of the near target, and Figs. 5(c) and (d) show the image and singular values of the distant target. The difference in the number of nonzero singular values between Figs. 5(b) and (d) confirms that the subspace of a distant target is narrower than that of a near target.

Another factor that can affect the target subspace dimension is the target size. For illustration, we simulate a square plate dihedral of two different areas: $0.09 \mathrm{~m}^{2}$ and $1 \mathrm{~m}^{2}$. The dihedral is placed at a standoff distance of $2.2 \mathrm{~m}$ from the radar without any wall. Figures 6(a) and (b) present the formed image and the normalized singular values of the small dihedral, and Figs. 6(c) and (d) show the formed image and the normalized singular values of the large dihedral. The difference in the number of nonzero singular values between Figs. 6(b) and (d) shows that the target subspace of the large dihedral is wider than that of the small dihedral.

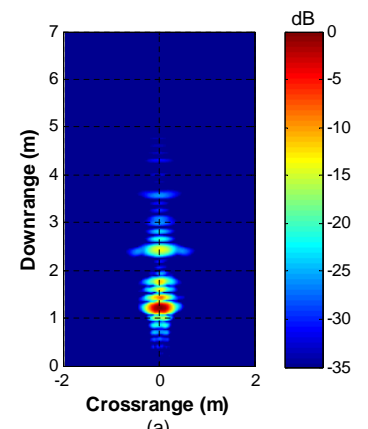

(a)

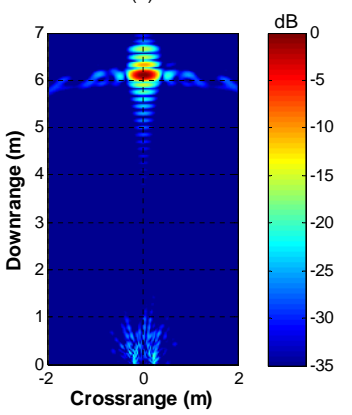

(c)

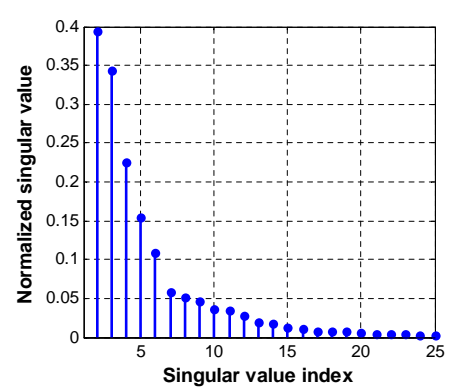

(b)

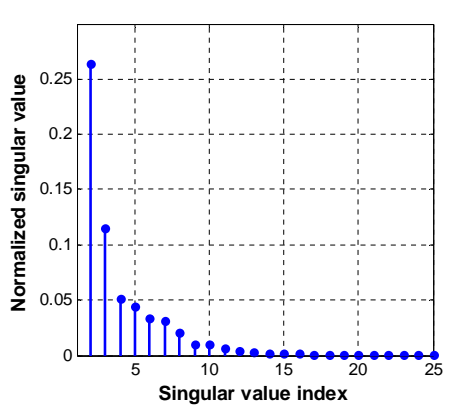

(d)
Fig. 5. Formed images and singular values of two different imaging ranges: (a) image of a near target, (b) its normalized singular values, (c) image of a distant target, and (d) its normalized singular values. For clarity, the first singular value is omitted; the other singular values are normalized with the first one.

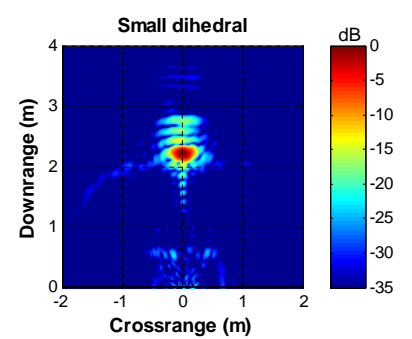

(a)

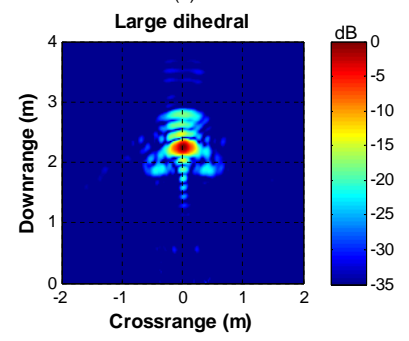

(c)

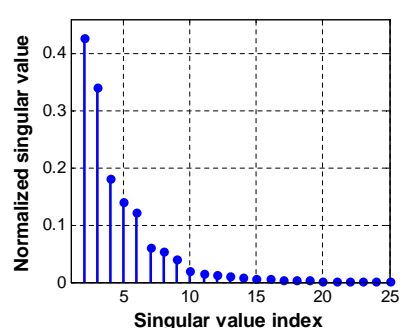

(b)

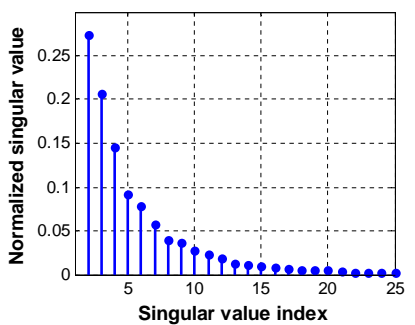

(d)
Fig. 6. Formed images and singular values of two different target sizes: (a) image of the small dihedral, (b) its normalized singular values, (c) image of the large dihedral, and (d) its normalized singular values. For clarity, the first singular value is omitted; the other singular values are normalized with the first one.

In summary, the target returns do not span a onedimensional subspace as reported in some existing literatures [12]-[14], but a multi-dimensional subspace, depending on several factors. These factors include, among others, the target location, the target size, the number of targets in the scene, and the antenna array configuration. Next, we investigate the eigen-structure of combined wall and target returns. 


\section{Combined Wall-Target Eigen-Subspace}

In the above analysis, we have shown that the wall subspace can be multi-dimensional and the target reflections span a multi-dimensional subspace. Here, we investigate the eigenstructure of combined wall and target returns. From a TWRI scene comprising a wall and target(s), the received signal can be expressed as a superposition of the wall and target returns

$$
s(m, n)=s_{\mathrm{w}}(m, n)+s_{\mathrm{t}}(m, n)+s_{\mathrm{wt}}(m, n),
$$

where $s_{\mathrm{w}}(m, n)$ is the wall returns, $s_{\mathrm{t}}(m, n)$ is the target returns, and $s_{\mathrm{wt}}(m, n)$ models the interactions (if they exist) between the target and wall. Since the wall reflections are relatively stronger than the behind-the-wall target reflections, it is assumed that the wall returns mostly lie in a subspace spanned by the singular vectors associated with the dominant singular values. Therefore, discarding the singular vectors associated with the dominant singular values can suppress the wall clutter in the formed image. For demonstration, we simulate a scene with two square plate dihedrals of area $0.16 \mathrm{~m}^{2}$ placed behind a homogeneous wall at coordinates $(-0.6,1.6) \mathrm{m}$ and $(0.6,1.3) \mathrm{m}$. The homogeneous wall has a thickness of $0.15 \mathrm{~m}$ and a dielectric constant of 7.6. Here, we slightly tilt the antenna array at an angle of two degree with respect to the wall surface to produce a multi-dimensional wall subspace. Using SVD, we decompose the signal matrix $\Phi$ into a set of $N$ singular components:

$$
\Phi=\Phi_{\mathrm{w}}+\Phi_{\mathrm{t}}+\Phi_{\mathrm{wt}}=\sum_{i=1}^{N} \sigma_{i} \boldsymbol{u}_{i} \boldsymbol{v}_{i}^{H} .
$$

where $\Phi_{\mathrm{wt}}$ is the signal matrix comprising the interactions between the wall and target(s). Figure 7 illustrates the formed images of the two targets behind the wall. Figure 7(a) shows the image formed by using all $N$ singular components in (25). Clearly, the wall reflections and ringing effects dominate the image and obscure the targets. Figure 7(b) presents the image after removing the first leading singular component, and Fig. 7(c) shows the image without the first two dominant singular components. Discarding just the first dominant singular component eliminates most of the wall reflections and the ringing effects. The target image in Fig. 7(c) is further enhanced by removing the second singular component.

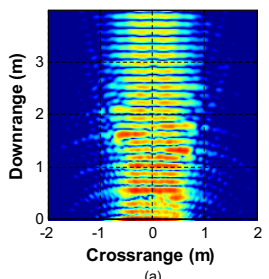

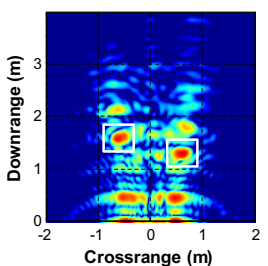

(b)

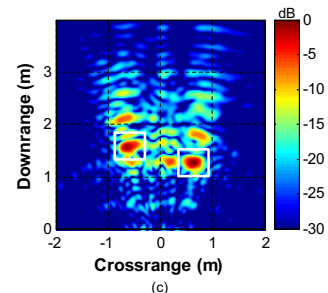

(c)
Fig. 7. Image of a scene with two dihedrals placed behind a homogeneous wall: (a) image of targets and wall, (b) image without the first dominant singular component, and (c) image without the first two leading singular components. The targets are circled by the rectangles.

Since the antenna array is not parallel to the wall surface, the wall subspace is spanned by several singular vectors. After the removal of the dominant singular components, the radar signal can still contain some wall residuals. These remaining wall returns are captured by singular vectors associated with non-dominant singular values, which interleave with the target singular values. To identify the singular vectors characterizing the wall returns, we propose a simple procedure. From (25), the matrix $\Phi$ consists of a weighted sum of $N$ singular components, where each singular component is given by the outer product of a pair of left and right singular vectors multiplied by its corresponding singular value. Let $\Psi_{i}$ denote the $i$-th singular component, given by

$$
\Psi_{i}=\sigma_{i} \boldsymbol{u}_{i} \boldsymbol{v}_{i}^{H}=\left[\boldsymbol{\psi}_{i 1}, \cdots, \boldsymbol{\psi}_{i N}\right],
$$

where $\psi_{i j}$ denotes the $j$-th column of the matrix $\Psi_{i}$. The range profile associated with the $i$-th singular component can be computed as

$$
\boldsymbol{r}_{i}=\frac{1}{N} \sum_{k=1}^{N} \operatorname{IFFT}\left(\boldsymbol{\psi}_{i k}\right),
$$

where IFFT denotes the inverse fast Fourier transform. The main peak in a range profile is used to indicate whether the singular component (SC) contains the wall or target returns, depending on the peak location with respect to the antenna standoff distance.

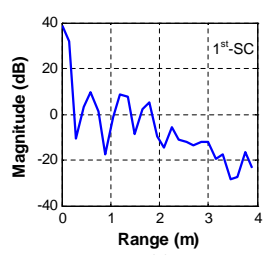

(a)

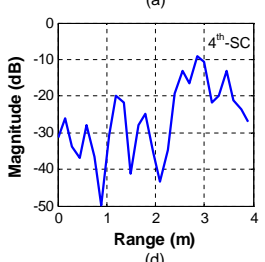

(d)

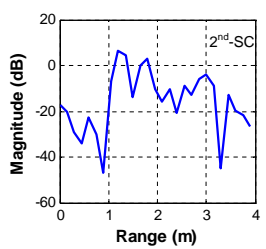

(b)

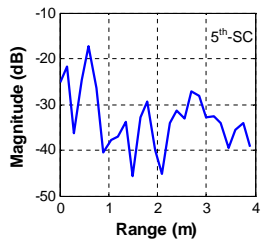

(e)
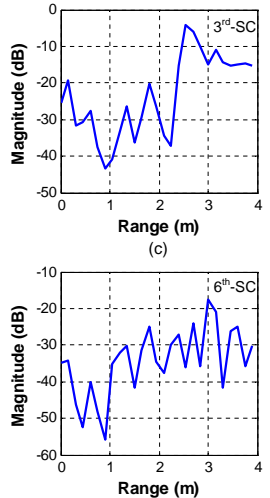

(f)
Fig. 8. The range profiles of the first 6 dominant singular components ( (1t $^{\mathrm{s}} \mathrm{SC}$ to $6^{\text {th }}-\mathrm{SC}$ ).

Figure 8 shows the range profiles of the first six singular components. The range profiles depicted in Figs. 8(a) and (b) show the first two singular components span the wall subspace as the distance of the main peak of their associated range profiles is less than the wall standoff distance. The range profiles in Figs. 8(c) and (d) associated with the third and fourth singular components have peaks beyond the wall standoff distance; these singular components span the target subspace. The small difference between the location of the peak in the range profile and the actual target range is due to the wall attenuation. Figure 8 (e) presents the range profiles of the fifth singular component $\left(5^{\text {th }}-\mathrm{SC}\right)$, indicating that some weak wall reflections reside in this singular component. Figure 9 presents images obtained from a subset of selected singular components. The image in Fig. 9(a) is reconstructed from the fifth singular component only and that depicted in Fig. 9(b) is obtained from the following subset of singular 
components: $3^{\text {rd }}, 4^{\text {th }}$, and $6^{\text {th }}-$ SCs. The simulation results show that apart from the first few dominant singular vectors, there are other non-dominant singular vectors that capture the wall returns. Though the non-dominant wall singular vectors interleave with the target singular vectors, their associated range profiles can be used to identify them. In the next section, we propose a technique to estimate the wall subspace and introduce a subspace projection method for mitigating the wall returns from the radar signals.
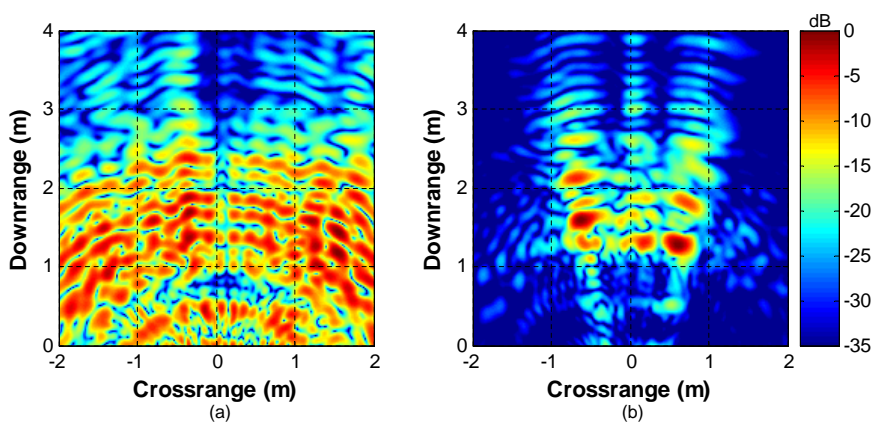

Fig. 9. Images formed using a subset of singular components: (a) image obtained from the $5^{\text {th }}$-SC only and (b) image obtained from the combination of these singular components: $3^{\text {rd }}-\mathrm{SC}, 4^{\text {th }}-\mathrm{SC}$, and $6^{\text {th }}-\mathrm{SC}$.

\section{Wall Clutter Mitigation Method}

The proposed wall clutter mitigation method is based on the assumption that the wall returns are relatively stronger than the target returns and they reside in separate subspaces. Therefore, SVD is used to decompose the signal matrix $\Phi$ as follows:

$$
\Phi=\sum_{i \in \mathcal{W}} \sigma_{i} \boldsymbol{u}_{i} \boldsymbol{v}_{i}^{H}+\sum_{i \in \mathcal{T}} \sigma_{i} \boldsymbol{u}_{i} \boldsymbol{v}_{i}^{H}+\sum_{i \in \mathcal{N}} \sigma_{i} \boldsymbol{u}_{i} \boldsymbol{v}_{i}^{H},
$$

where $\mathcal{W}, \mathcal{T}$, and $\mathcal{N}$ are the sets of indices for wall, target, and noise singular vectors, respectively. However, not all wall singular components will be associated with the dominant singular values. While it is expected that the strong wall reflections will be represented by the first few singular vectors associated with the dominant singular values, some weak components of the wall returns may reside in a subspace spanned by other singular vectors associated with non-dominant singular values. Therefore, we propose a method for estimating the wall subspace followed by a subspace projection method for mitigating the wall returns from the radar signals.

\section{A. Wall Subspace Estimation}

The proposed estimation method for wall subspace is based on the assumption that the strong reflections from the front and back of the wall are captured by the first few dominant singular components. In [29], similar assumption was made to estimate the time delay of ultra-wideband radar signals backscattered from a wall. First, we estimate the wall range, i.e., the distance from the antenna to the back of the wall from the range profiles of the dominant singular components. Then, we classify the remaining singular components into the wall and target classes based on their range profiles. The indices of the singular vectors forming the wall singular components are stored in the index set $\mathcal{W}$. Let $\eta$ denote the wall range. When the standoff distance $z_{a}$ and the wall thickness $d$ are known, the wall range can be approximated as

$$
\eta \approx\left(d \sqrt{\epsilon}+z_{a}\right)
$$

In practice, the exact values of the wall parameters are not readily available. Therefore, we determine the wall range from the range profiles associated with the dominant singular vectors. To determine the leading singular vectors associated with the wall, we apply a threshold technique to segment the singular value spectrum into two classes, one of which is the dominant wall singular values. Suppose the range of singular values is $\left[0, \sigma_{\max }\right]$. Given a threshold $\delta \in\left[0, \sigma_{\max }\right]$, the singular value spectrum can be partitioned into two classes: $\mathcal{C}_{\mathrm{w}}=\left\{\sigma_{i} \geq \delta\right\}$ and $\mathcal{C}_{\mathrm{t}}=\left\{\sigma_{i}<\delta\right\}$. Here, we employ Otsu's method [30], which computes the optimum threshold $\widehat{\delta}$ by maximizing the between-class variance:

$$
\Sigma_{0}=\mathcal{P}_{\mathrm{w}}\left(\mu_{\mathrm{w}}-\mu_{0}\right)^{2}+\mathcal{P}_{\mathrm{t}}\left(\mu_{\mathrm{t}}-\mu_{0}\right)^{2},
$$

where $\mathcal{P}_{\mathrm{w}}$ and $\mathcal{P}_{\mathrm{t}}$ are the class probabilities, $\mu_{\mathrm{w}}$ and $\mu_{\mathrm{t}}$ are the class means, and $\mu_{0}$ is the total mean of the classes. For more details on how to determine the optimum threshold of Otsu's method, the interested reader is referred to Appendix A.

Then, Eq. (27) is used to compute the range profiles associated with the singular values in the class $\mathcal{C}_{\mathrm{w}}$. Let $h_{i}$ denote the distance of the main peak in the range profile associated with the $i$-th singular value belonging to the wall. The wall range $\eta$ can be estimated as

$$
\eta=\max _{i}\left(h_{i}\right)
$$

From the estimated wall range, we can now identify the remaining wall singular components and determine the singular vectors spanning the wall subspace. We classify a singular vector spanning the wall subspace when the main peak of its associated range profile is located inside the wall range $\eta$. This classification is performed on all singular components of $\Phi$ and the indices of the wall singular vectors are stored in the index set $\mathcal{W}$.

\section{B. Wall Clutter Mitigation}

After identifying the wall subspace, we remove the wall returns by projecting the radar signals onto the subspace orthogonal to the wall subspace. Similarly, the noise can be removed by projecting the radar signals onto the subspace orthogonal to the noise subspace. First, the radar signal is preprocessed to remove the common signal across the array aperture. Let $\widetilde{\Phi}$ be the matrix obtained after subtracting the mean vector from each column of $\Phi$,

$$
\widetilde{\Phi}=\Phi-\boldsymbol{m} \boldsymbol{e}^{T},
$$

where $\boldsymbol{m}$ is the mean of the columns of $\Phi$ and $\mathbf{e}^{T}=[1, \ldots, 1]$, $e \in R^{N}$. Using SVD, we decompose the matrix $\widetilde{\Phi}$ as

$$
\widetilde{\Phi}=\widetilde{U} \widetilde{\Sigma} \widetilde{V}^{H}
$$

where $\widetilde{U}=\left[\widetilde{\boldsymbol{u}}_{1}, \ldots, \widetilde{\boldsymbol{u}}_{M}\right], \widetilde{V}=\left[\widetilde{\boldsymbol{v}}_{1}, \ldots, \widetilde{\boldsymbol{v}}_{N}\right]$, and $\widetilde{\Sigma}_{i, i}=\widetilde{\sigma}_{i}$. Summing the outer product of the pair of singular vectors in 
the index set $\mathcal{W}$ generates the wall subspace, which is given by

$$
P_{\mathrm{w}}=\sum_{i \in \mathcal{W}} \widetilde{\boldsymbol{u}}_{i} \widetilde{\boldsymbol{v}}_{i}^{H}
$$

The subspace orthogonal to the wall subspace is computed as

$$
P_{\mathrm{w}}^{\perp}=I-P_{\mathrm{w}} P_{\mathrm{w}}^{H},
$$

where $I$ denotes the identity matrix. To mitigate the wall returns, the matrix $\widetilde{\Phi}$ is projected onto the orthogonal subspace:

$$
\widehat{\Phi}=P_{\mathrm{w}}^{\perp} \widetilde{\Phi} .
$$

The resulting matrix $\widehat{\Phi}$ is further processed to remove noise. The subspace orthogonal to the noise subspace can be expressed as

$$
P_{\mathrm{n}}^{\perp}=I-P_{\mathrm{n}} P_{\mathrm{n}}^{H},
$$

where $P_{\mathrm{n}}=\sum_{i \in \mathcal{N}} \widehat{\boldsymbol{u}}_{i} \widehat{\boldsymbol{v}}_{i}^{H}$ is the noise subspace. The pair of left and right singular vectors, i.e., $\widehat{\boldsymbol{u}}$ and $\widehat{\boldsymbol{v}}$ are obtained from the SVD of $\widehat{\Phi}$. Since noise is characterized by singular vectors associated with small singular values, there are several methods to determine the noise subspace. Akaike Information Criterion (AIC) and Minimum Description Length (MDL) methods are two commonly used methods to estimate the noise subspace [15]. The AIC is given by

$$
\operatorname{AIC}(i)=N \log \frac{\left((1 /(M-i)) \sum_{m=i+1}^{M} \sigma_{m}\right)^{M-i}}{\prod_{m=i+1}^{M} \sigma_{m}}+
$$

where $\sigma_{i}$ is the $i$-th singular value of $\widehat{\Phi}$. Similarly, the MDL is given by

$$
\operatorname{MDL}(i)=N \log \frac{\left((1 /(M-i)) \sum_{m=i+1}^{M} \sigma_{m}\right)^{M-i}}{\prod_{m=i+1}^{M} \sigma_{m}}+
$$

The number of singular values belonging to the noise class is determined by minimizing the AIC or MDL. Once the wall and noise subspaces are computed, the new matrix $\bar{\Phi}$, which contains the target reflections, is written as

$$
\bar{\Phi}=P_{\mathrm{n}}^{\perp}\left(P_{\mathrm{w}}^{\perp} \widetilde{\Phi}\right)=P_{\mathrm{t}} \widetilde{\Phi},
$$

where $P_{\mathrm{t}}=P_{\mathrm{n}}^{\perp} P_{\mathrm{w}}^{\perp}$ is the target subspace projection operator. Finally, DS beamforming is applied to signals of $\bar{\Phi}$ to form an image of the scene.

The proposed method is initially tested on the simulated data obtained from the scene with two dihedrals placed behind a homogeneous wall. To measure the performance of the wall clutter mitigation method, we compute the improvement factor (IF) in terms of the target-to-clutter ratio (TCR)

$$
\mathrm{IF}=10 \log \left(\frac{\mathrm{TCR}_{o}}{\mathrm{TCR}_{i}}\right),
$$

where $\mathrm{TCR}_{o}$ and $\mathrm{TCR}_{i}$ are, respectively, the target-to-clutter ratios of the formed image with and without the use of a wall clutter mitigation method. The TCR of a radar image is calculated as

$$
\mathrm{TCR}=\frac{\frac{1}{N_{t}} \sum_{(x, z) \in A_{t}}|I(x, z)|^{2}}{\frac{1}{N_{c}} \sum_{(x, z) \in A_{c}}|I(x, z)|^{2}},
$$

where $A_{t}$ is the target region, $A_{c}$ is the clutter region defined as the entire image excluding the target region, $N_{c}$ and $N_{t}$ are, respectively, the number of pixels in the clutter and target regions. The quality of the target image is measured in terms of the target power ratio (TPR) given by

$$
\mathrm{TPR}=\frac{\mathbf{P}_{j}}{\mathbf{P}_{0}},
$$

where $\quad \mathbf{P}_{0}=\frac{1}{N_{t}} \sum_{(x, z) \in A_{t}}\left|I_{0}(x, z)\right|^{2}, \quad \mathbf{P}_{j}=$ $\frac{1}{N_{t}} \sum_{(x, z) \in A_{t}}\left|I_{j}(x, z)\right|^{2}$, and $I_{0}(x, z)$ and $I_{j}(x, z)$ denote, respectively, the formed image after background substraction and the formed image after removing the first $j$ dominant singular components from the matrix $\Phi$. Figure 10 shows images formed by DS beamforming before and after wall clutter mitigation. Without wall clutter mitigation, Fig. 10(a) shows an image with strong clutter. With background subtraction, the formed image shown in Fig. 10(b) is free of wall clutter; both targets are clearly visible. However, in practice, it is difficult to have access to the measurements of the background scene devoid of targets. Figure 10(c) depicts the image obtained with the proposed method, where the wall clutter is markedly suppressed. Table I lists the improvement factor (IF) and the target power ratio of the formed images after wall clutter mitigation. Background subtraction obtains the highest IF of $11.14 \mathrm{~dB}$, followed by the proposed subspace projection method with an IF of $10.09 \mathrm{~dB}$. In terms of TPR, the proposed method achieves a TPR of $-3.06 \mathrm{~dB}$. When the dominant singular components are removed without the use of the proposed wall subspace estimation method, the IFs of the formed images are presented as follows. After the removal of the dominant singular component from the matrix $\Phi$, the IF of the formed image is $4.89 \mathrm{~dB}$. Discarding the first two leading singular components improves the IF of the image to $9.98 \mathrm{~dB}$. However, when we remove the first three singular components from the matrix $\Phi$, the IF of the formed images decreases slightly to $7.32 \mathrm{~dB}$. The TPR of the formed target image also decreases markedly when discarding the first three singular components.

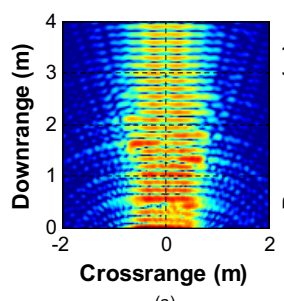

(a)

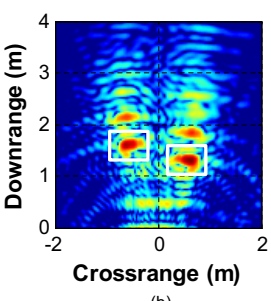

(b)

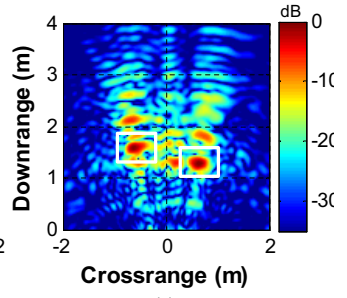

(c)
Fig. 10. Images before and after wall clutter mitigation: (a) image formed without wall clutter mitigation, (b) image obtained after using background subtraction, and (c) image obtained with the proposed method. 
TABLE I

IMPROVEMENT FACTOR OF THE IMAGE PRODUCED BY THE PROPOSED SUBSPACE PROJECTION METHOD AND THE BASIC SVD-BASED METHOD WITH THE REMOVAL OF DOMINANT SINGULAR COMPONENTS, TESTED ON SYNTHETIC DATA.

\begin{tabular}{|l|r|r|}
\hline & \multicolumn{1}{|c|}{ IF } & TPR \\
\hline Background subtraction & $11.14 \mathrm{~dB}$ & $0 \mathrm{~dB}$ \\
\hline Proposed wall clutter mitigation method & $10.09 \mathrm{~dB}$ & $-3.06 \mathrm{~dB}$ \\
\hline Removal of the first singular component & $4.89 \mathrm{~dB}$ & $-0.48 \mathrm{~dB}$ \\
\hline Removal of the first two singular components & $9.98 \mathrm{~dB}$ & $-2.97 \mathrm{~dB}$ \\
\hline Removal of the first three singular components & $7.32 \mathrm{~dB}$ & $-8.06 \mathrm{~dB}$ \\
\hline
\end{tabular}

So far the proposed wall clutter mitigation technique has been applied to a noiseless TWRI scene. The proposed method is further tested under different noise levels, where the simulated radar signals are corrupted by additive white Gaussian noise. The IF of the image formed by the proposed method is computed as a function of the signal-to-noise ratio (SNR) of the input signal. Figure 11 illustrates the variations in the IF of the formed image as a function of the SNR of the input signal. The IF of the formed image remains unchanged until the SNR of the input signal decreases to $40 \mathrm{~dB}$. Figure 12 shows examples of radar images obtained from input signal with SNR of $20 \mathrm{~dB}$ and $40 \mathrm{~dB}$. In the next section, the subspace projection method is evaluated on real radar data collected from a ground-based stepped-frequency TWRI system.

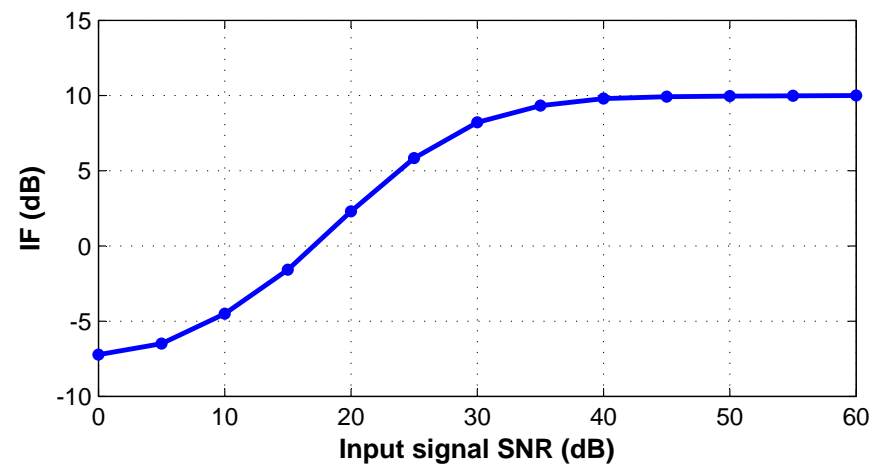

Fig. 11. Improvement factor of the image as a function of the SNR of the input radar signal.

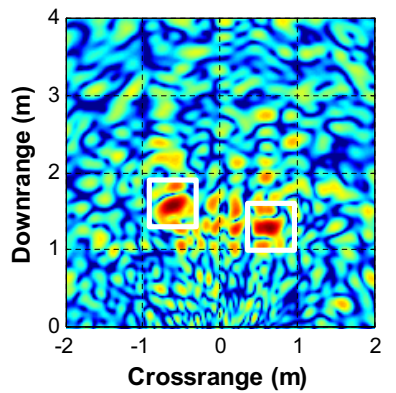

(a)

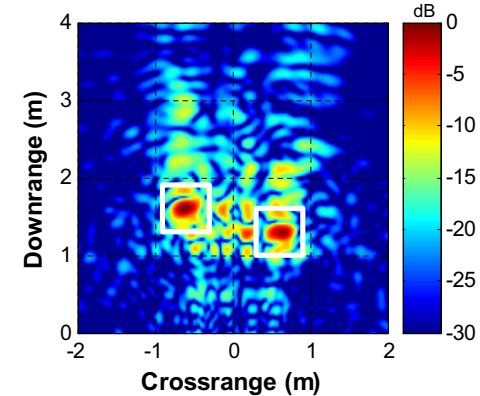

(b)
Fig. 12. Examples of images obtained from input signal of different SNRs: (a) images formed using input signal with SNR of $20 \mathrm{~dB}$ and (b) image formed using input signal with SNR of $40 \mathrm{~dB}$.

\section{EXPERIMENTAL RESULTS}

Real radar signals are collected in the Radar Imaging Lab of the Center for Advanced Communications at Villanova University, PA, USA. An Agilent network analyzer, Model ENA 5017B, is used to implement a stepped-frequency waveform for synthesizing a one-dimensional (1-D) and a twodimensional (2-D) array apertures. A 7.62 by 7.62 meter room with pyramidal foam and laminated polyurethane foam sheet absorbers on the side and back walls is constructed for imaging. For more details about the room setting and the specification of the radar system, the interested reader is referred to [6].

\section{A. Experimental Setup}

For evaluation purposes, a 1-D and 2-D synthesized array apertures are used for 2-D and 3-D TWRI, respectively. Furthermore, two different TWRI scenarios are designed using two types of walls: a $0.14 \mathrm{~m}$ thick solid concrete wall and a $0.127 \mathrm{~m}$ thick hollow drywall. The drywall is built from a wooden frame, which is fastened with $0.019 \mathrm{~m}$ plywood on one side and $0.016 \mathrm{~m}$ gypsum wallboard on the other side. In the first scenario, the radar is placed at a standoff distance of $1.16 \mathrm{~m}$ from the concrete wall and a dihedral is placed at $2.1 \mathrm{~m}$ behind the wall. An array aperture of length 1.2446 $\mathrm{m}$ is synthesized with $0.0222 \mathrm{~m}$ inter-element spacing, and a stepped-frequency signal covering 0.7 to $3.1 \mathrm{GHz}$ frequency band is used to interrogate the scene. The second scenario involves a scene populated with nine targets of different RCS placed behind the drywall. Figure 13 shows a picture of the second scene and its ground-truth. The nine targets in the second scene are three dihedrals, four trihedrals, a sphere and a tophat. Each target is located at a certain height and position, as shown in Fig. 13(a). Its location within the scene is given in the ground-truth image depicted in Fig. 13(b). A 69-antenna array of length $1.5 \mathrm{~m}$ is used to interrogate the scene. The stepped-frequency signal has a bandwidth of $1 \mathrm{GHz}$ centered at $2.5 \mathrm{GHz}$. Table II lists the characteristics of the reflectors used in the two TWRI scenes.

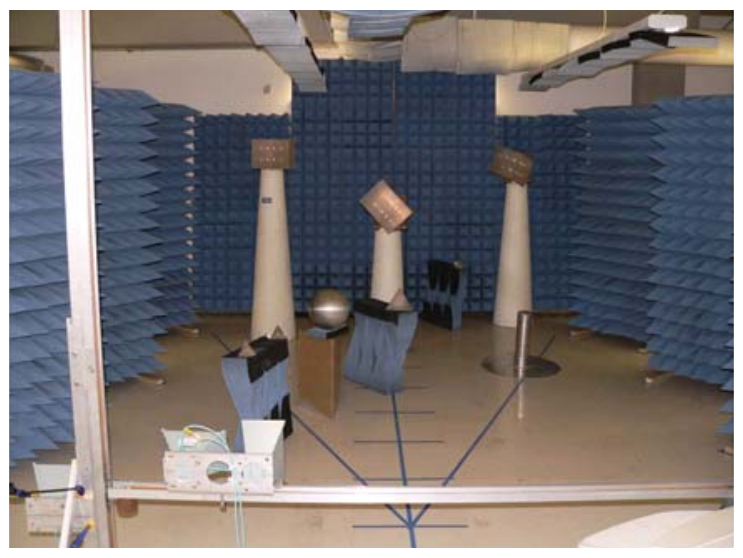

(a) 


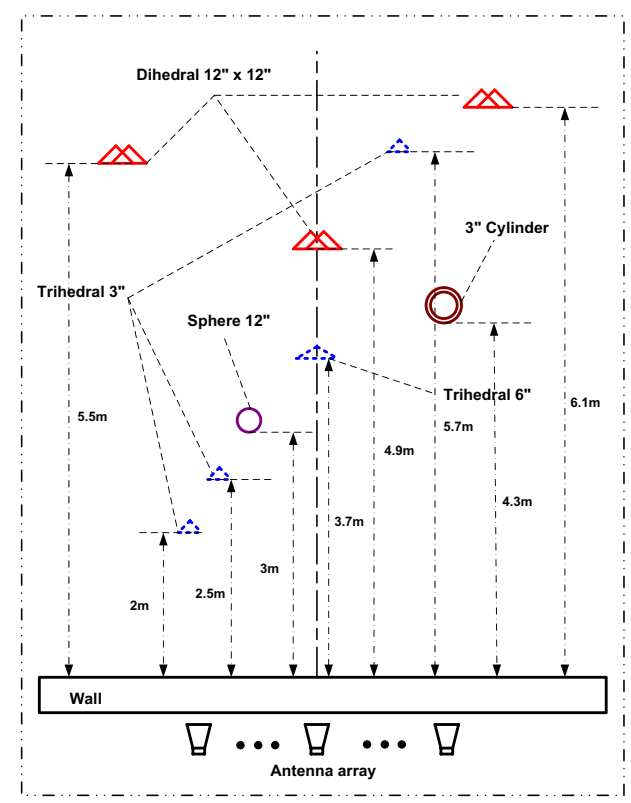

(b)

Fig. 13. Picture of the drywall scene: (a) image depicting the nine targets and (b) the ground truth image.

TABLE II

REFLECTORS USED IN THE TWRI EXPERIMENTS

\begin{tabular}{|l|}
\hline $0.0762 \mathrm{~m}$ seam triangular plate trihedrals, $\mathrm{RCS}=-20.6 \mathrm{dBsm}$ \\
\hline $0.1524 \mathrm{~m}$ seam triangular plate trihedrals, $\mathrm{RCS}=-8.5 \mathrm{dBsm}$ \\
\hline $0.3048 \mathrm{~m}$ square plate dihedrals, $\mathrm{RCS}=11.3 \mathrm{dBsm}$ \\
\hline $0.3048 \mathrm{~m}$ diameter sphere, $\mathrm{RCS}=-11.4 \mathrm{dBsm}$ \\
\hline Tophat: $0.127 \mathrm{~m}$ cylinder width, $0.508 \mathrm{~m}$ cylinder height, \\
$0.711 \mathrm{~m}$ circular ground plane diameter \\
\hline
\end{tabular}

\section{B. Two-Dimensional Through the Wall Radar Imaging}

A line array aperture is synthesized for the above described scenes to perform 2-D imaging. Before DS beamforming, four different methods are used for wall clutter mitigation: background subtraction, time gating, spatial filtering, and Bscanbased SVD methods [12]-[15]. In [12]-[14], SVD is applied to the B-scan, which is obtained by applying inverse fast Fourier transform on the space-frequency measurements. The first and second dominant singular components are assumed to contain the wall and target returns, respectively. In [15], SVD is used to decompose the formed image into a set of eigenimages. For wall clutter mitigation, the first dominant eigenimage is discarded. Then, an information theoretic criteria method is used to determine the eigen-images spanning the target subspace. In time gating, the stepped-frequency signal is transformed into a range profile. Based on the standoff distance and the wall parameters, the radar returns corresponding to the wall region are set to zero and the range profile is converted back to the frequency domain. For background subtraction, radar signals from an empty scene devoid of target(s) are subtracted from the radar signals received from the scene populated with target(s) before DS beamforming is applied to reconstruct the image. Background subtraction represents an ideal scenario, where access to the background scene is available; this is not possible in real scenarios. In spatial filtering, an infinite-impluse notch filter is used to remove zero frequency component. The frequency response of the notch filter is defined as

$$
H(j \omega)=\frac{1-\exp (-j \omega)}{1-a \exp (-j \omega)},
$$

where $\omega$ is the angular frequency and $a(<1)$ is a positive constant denoting the width of the filter notch. In our experiments, we define $a$ at the point of achieving maximum IF. The concrete wall scene, which has a dihedral, is illuminated by the synthesized array aperture, producing a signal matrix $\Phi$ of size $801 \times 57$, i.e., 801 frequencies and 57 antennas. All five wall clutter mitigation approaches including the proposed subspace projection method are used to suppress the wall clutter in the formed image.

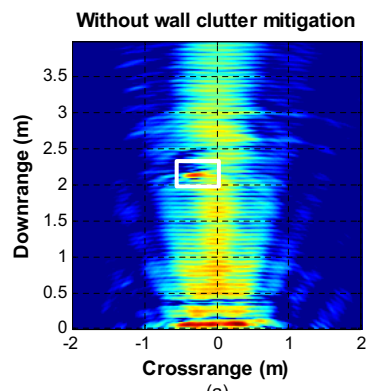

(a)

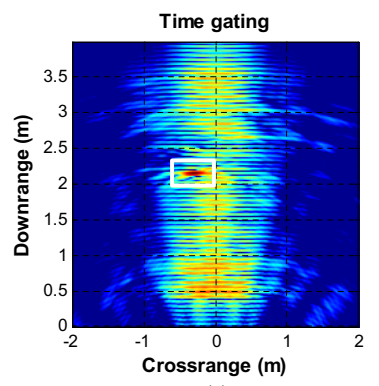

(c)

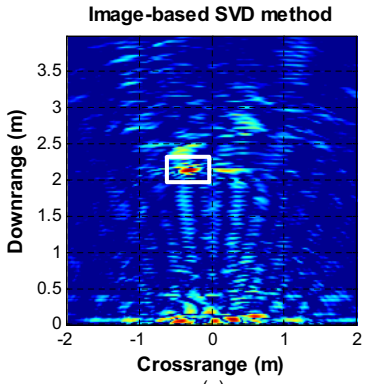

(e)

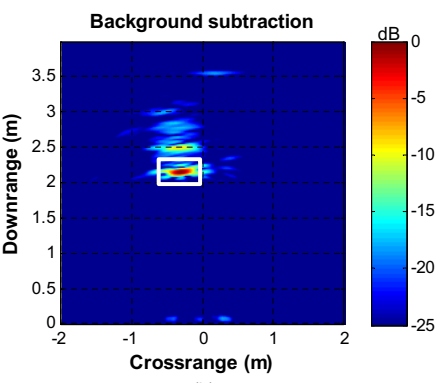

(b)
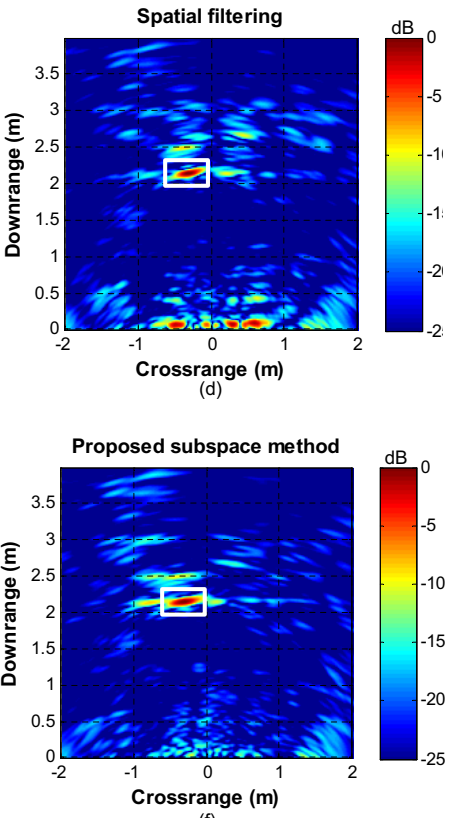

(f)
Fig. 14. Image of the concrete wall scene obtained using different wall clutter mitigation methods: (a) no wall clutter mitigation, (b) background subtraction, (c) time gating, (d) spatial filtering, (e) image-based SVD method, and (f) proposed subspace projection method.

Figure 14 illustrates images before and after wall clutter mitigation, using different wall mitigation methods. With the availability of the background measurements, background subtraction produces a clear image, Fig. 14(b), in which most of the wall and background clutter is removed. With time gating, 
the formed image contains strong wall clutter, see Fig. 14(c). Even though the target is far from the wall, time gating cannot suppress the wall clutter because the wall reverberations and target reflections highly overlap in the time domain. The image in Fig. 14(d) shows spatial filtering is effective in removing the wall reflections without significantly compromising the target image. The image-based SVD method [15] produces an image where most of the wall clutter is suppressed but the shape of the target is distorted compared to that shown in Fig. 14(b), obtained using background subtraction. Figure 14(f) illustrates the image produced by the proposed subspace projection method. This image does not contain the wall clutter and is as clear as that of the spatial filtering method. Figure 15 depicts the wall and target singular values identified by the proposed wall subspace estimation method. The singular values depicted in Fig. 15(a) and (b) belong to the wall and target, respectively. From Fig. 15(a), we can see that the wall subspace comprises the first two dominant singular components and components 5, 23, 24, and 25. It is clear from Fig. 15(a) that the non-dominant wall singular values interleave with the target singular values. Table III presents the improvement factor (IF) in terms of TCR of the wall clutter mitigation methods for the images shown in Fig. 14. Background subtraction achieves the highest IF of $16.16 \mathrm{~dB}$, followed by the proposed subspace projection method with an IF of $11.01 \mathrm{~dB}$. Spatial filtering gives an IF of $8.26 \mathrm{~dB}$. Among the SVD methods, the image-based SVD method give better result than the Bscan-based SVD method as it assumes that the target reflections reside in a multi-dimensional subspace.
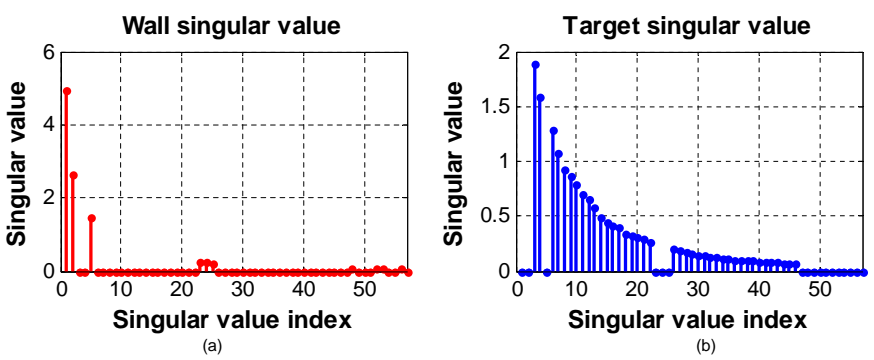

Fig. 15. Singular value spectrum of (a) the wall and (b) the target subspaces as identified by proposed wall subspace estimation method for the scene with a dihedral behind the concrete wall.

TABLE III

IMPROVEMENT FACTOR OF THE WALL MITIGATION METHODS TESTED ON RADAR DATA COLLECTED FROM THE CONCRETE WALL SCENE.

\begin{tabular}{|l|c|}
\hline Wall clutter mitigation method & Improvement factor (IF) \\
\hline Proposed subspace projection method & $11.01 \mathrm{~dB}$ \\
\hline Background subtraction & $16.16 \mathrm{~dB}$ \\
\hline Time gating & $3.00 \mathrm{~dB}$ \\
\hline Spatial filtering [9] & $8.26 \mathrm{~dB}$ \\
\hline Bscan-based SVD method [14] & $2.84 \mathrm{~dB}$ \\
\hline Image-based SVD method with AIC [15] & $6.30 \mathrm{~dB}$ \\
\hline Image-based SVD method with MDL [15] & $6.30 \mathrm{~dB}$ \\
\hline
\end{tabular}

For the drywall scene, Fig. 16 depicts the formed images before and after wall clutter mitigation. Without any preprocessing, Fig. 16(a) depicts an image with strong wall clutter.

With the availability of an empty scene, background subtraction produces a clear radar image, Fig. 16(b). Time gating and spatial filtering fail to remove the wall contributions from the radar data, Figs. 16(c) and (d). The SVD methods can hardly suppress the wall clutter in the formed radar image. This is because the wall reflections is assumed to reside in the first singular component only. However, the reflections backscattered from a heterogeneous wall span a multi-dimensional subspace space as described in Section III-B. Figure 16(e) shows the output image of the image-based SVD method with AIC; only the targets with large RCS are barely visible. The Bscan-based SVD methods perform poorly because the wall subspace is assumed to be one dimensional. Figure 16(f) shows the image obtained using the proposed wall clutter mitigation method, where most of the wall clutter is significantly suppressed. Table IV lists the IF of the images presented in Fig. 16. The proposed method achieves the second highest IF of $22.35 \mathrm{~dB}$. The Bscan-based SVD method described in [14] gives the lowest IF of $2.08 \mathrm{~dB}$. The experimental results demonstrate that the proposed method can be as effective as background subtraction in removing the clutter due to both homogeneous and heterogeneous walls. In the next section, we apply the proposed wall clutter mitigation method to 3-D TWRI.

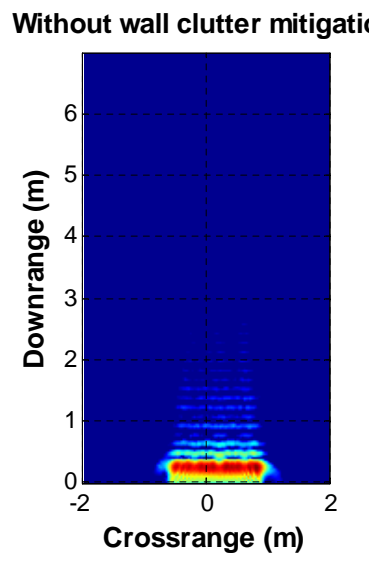

(a)

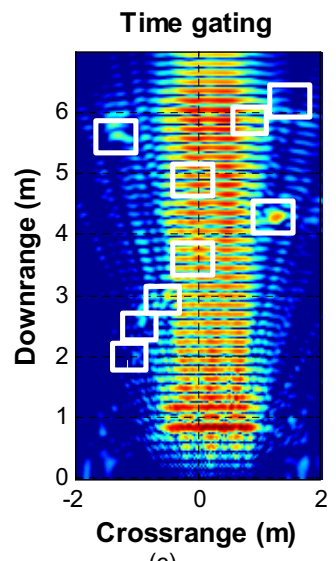

(c)

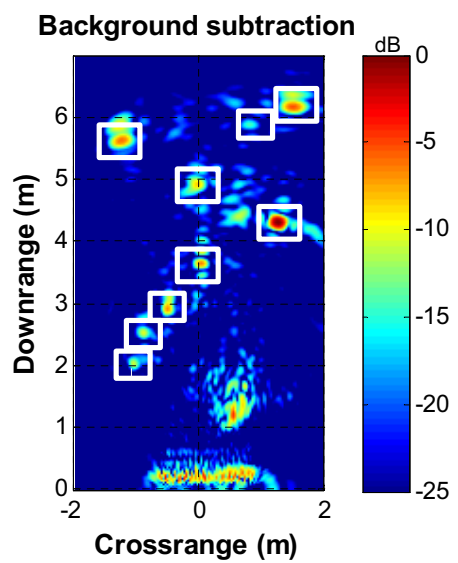

(b)

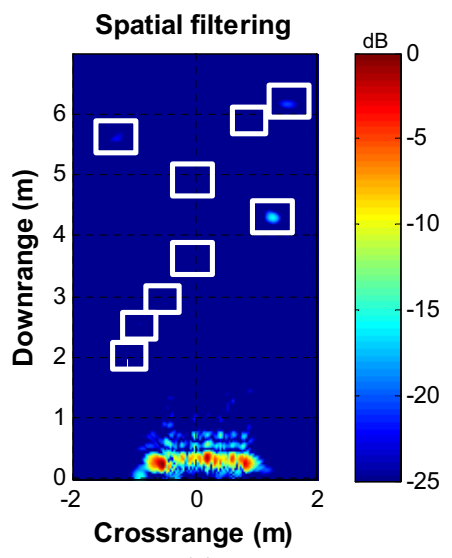

(d) 


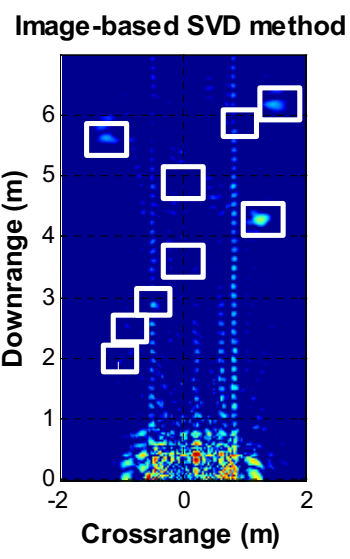

(e)

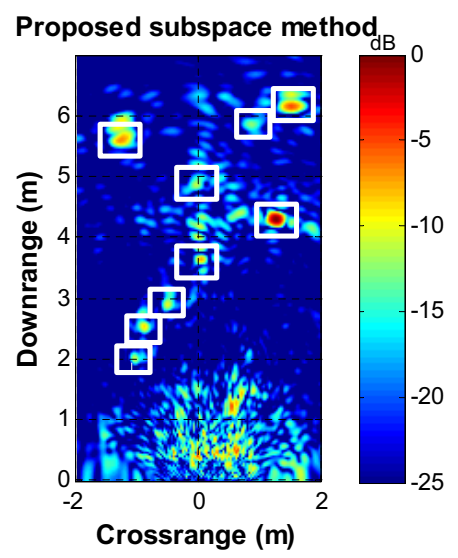

(f)
Fig. 16. Image of the drywall scene obtained using different wall clutter mitigation method: (a) no wall clutter mitigation, (b) background subtraction, (c) time gating, (d) spatial filtering, (e) image-based SVD method and (f) proposed subspace projection method.

TABLE IV

IMPROVEMENT FACTOR OF WALL MITIGATION METHODS BASED ON THE SCENE WITH NINE TARGETS BEHIND THE DRYWALL.

\begin{tabular}{|l|c|}
\hline Wall Clutter Mitigation Approach & Improvement factor (IF) \\
\hline Proposed subspace projection method & $22.35 \mathrm{~dB}$ \\
\hline Background subtraction & $24.50 \mathrm{~dB}$ \\
\hline Time gating & $13.60 \mathrm{~dB}$ \\
\hline Spatial filtering [9] & $10.24 \mathrm{~dB}$ \\
\hline Bscan-based SVD method [14] & $2.08 \mathrm{~dB}$ \\
\hline Image-based SVD method with AIC [15] & $13.66 \mathrm{~dB}$ \\
\hline Image-based SVD method with MDL [15] & $13.65 \mathrm{~dB}$ \\
\hline
\end{tabular}

\section{Three-Dimensional Through the Wall Radar Imaging}

For 3-D imaging, the scene is scanned by a 2-D array aperture along the horizontal and vertical directions to reveal the properties of targets residing behind the wall, e.g., the height of the target. The received monochromatic signals for all $M$ frequencies at each antenna location of the 2-D array aperture are stacked to form a column of signal matrix $\Phi \in$ $\mathcal{C}^{M \times N}$, where $N$ is the number of antenna locations in the 2-D array aperture. The order of selecting the antenna locations, i.e., processing row-wise or column-wise, only results in a permutation of the columns of $\Phi$. It can be readily shown that the permutation of the columns of the matrix $\Phi$ does not change the column order of the left and right singular vectors, and more importantly, it does not affect the singular values. Hence, the arrangement of the received signals into a matrix $\Phi$ does not affect the wall and target subspaces. To form 3-D images, DS beamforming is applied to compute the complex amplitude of each voxel $I(z, x, y)$ :

$$
I(x, z, y)=\frac{1}{N M} \sum_{n=0}^{N-1} \sum_{m=0}^{M-1} s(m, n) \exp \left(j \omega_{m} \tau_{n}(x, z, y)\right),
$$

where $\tau_{n}(x, z, y)$ is the focusing delay from the $n$-th antenna of the 2-D array aperture to the voxel at location $(x, z, y)$. The computation of the two-way propagation delay from an antenna to a voxel is described in [8].

A 2-D array aperture is used to interrogate the concrete wall and drywall scenes. Background subtraction and the proposed subspace projection method are then applied to mitigate the wall returns. We should point out that for the sake of clarity, the voxels below $-25 \mathrm{~dB}$ were thresholded in the 3-D images. Figure 17 illustrates the 3-D radar images of the concrete wall scene. Applying DS beamforming directly to the space-frequency measurements produces a cluttered 3-D image, Fig. 17(a). Figure 17(b) shows the image obtained from background subtraction which does not have wall clutter. The image shown in Fig. 17(c), which is produced by the proposed wall clutter mitigation method, is as clear as that produced by background subtraction. For the drywall scene, the formed images obtained using background subtraction and the proposed method are shown in Fig. 18; both images are free of wall clutter. Table V presents the IF of the thresholded 3-D images depicted in Figs. 17 and 18. The proposed subspace projection method gives an IF of $21.33 \mathrm{~dB}$ for the concrete wall scene and $25.26 \mathrm{~dB}$ for the drywall scene, compared to background subtraction, which yields IF values of $20.83 \mathrm{~dB}$ and $25.06 \mathrm{~dB}$, respectively.
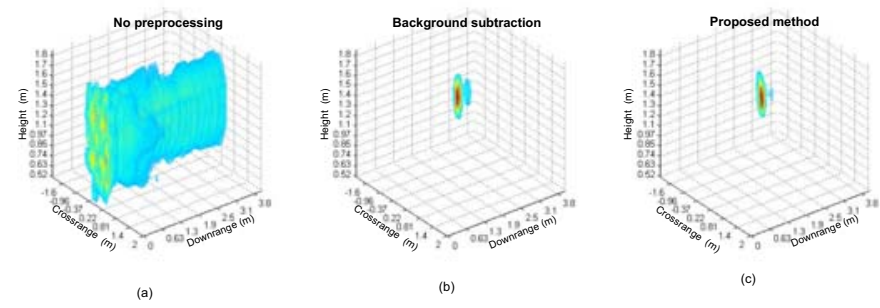

Fig. 17. The 3-D images of the concrete wall scene: (a) before wall clutter mitigation, after the use of (b) background subtraction, and (c) the proposed subspace projection method. For visualization, the 3-D images are displayed in linear scale and voxels less than $-25 \mathrm{~dB}$ are removed.
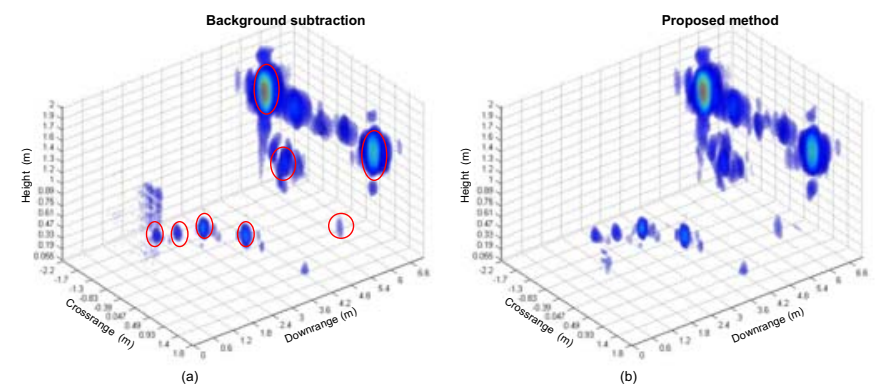

Fig. 18. The 3-D images of the drywall scene after the use of (a) background subtraction and (b) the proposed subspace projection method. For visualization, the 3D images are displayed in linear scale and voxels less than -25 $\mathrm{dB}$ are removed.

\section{CONCLUSION}

Strong signal reflections from the exterior wall hinder the visibility of stationary targets in through-the-wall radar imaging. This paper presented a comprehensive analysis of the eigen-structure of imaged TWRI scenes. The analysis showed 
TABLE V

IMPROVEMENT FACTOR OF THE WALL CLUTTER MITIGATION METHODS FOR 3-D IMAGING.

\begin{tabular}{|l|c|c|}
\hline Approach & concrete wall scene & drywall scene \\
\hline Proposed method & $21.33 \mathrm{~dB}$ & $25.26 \mathrm{~dB}$ \\
\hline Background subtraction & $20.83 \mathrm{~dB}$ & $25.06 \mathrm{~dB}$ \\
\hline
\end{tabular}

that when the radar is placed parallel to a homogeneous wall of uniform thickness, the wall returns span a one-dimensional subspace. However, when the antenna is not perfectly aligned with the wall surface or the wall thickness is not uniform, which is often the case in practice, the wall reflections span a multi-dimensional subspace. For a heterogeneous walls, the wall returns also span a multi-dimensional subspace. Furthermore, the analysis showed that the target subspace spanned by several singular vectors, depending on the target location, target size, number of targets in the scene, and the configuration of the antenna array.

For wall clutter mitigation, we proposed a method that estimates the wall subspace and a subspace projection approach to remove, or at least significantly suppress, the wall clutter. The proposed approach does not assume prior knowledge of the scene nor the wall electromagnetic characteristics. It was applied to mitigate wall clutter in 2-D and 3-D TWRI. Experiments with simulated and real data showed that the proposed method was as effective as background subtraction in removing wall clutter and revealing the behind-the-wall targets-without prior knowledge of the background scene.

\section{APPENDIX A}

\section{OTsu Thresholding Method}

Suppose we have $N$ singular values which lie in the range $\left[0, \sigma_{\max }\right]$, and the spectrum of singular values is divided into $L$ equal intervals $\left[\xi_{i}, \xi_{i+1}\right)$, for $i=0, \ldots, L-1$. Let $\mathcal{P}\left(\xi_{i}\right)$ denote the probability mass defined by the relative frequency of singular values in the $i$-th interval; that is,

$$
\mathcal{P}\left(\xi_{i}\right)=n\left(\xi_{i}\right) / N
$$

where $n\left(\xi_{i}\right)$ is the number of singular values $\sigma_{i} \in\left[\xi_{i}, \xi_{i+1}\right)$. For a given threshold $\tau=\xi_{k}, k=0, \ldots, L-1$, the spectrum of singular values can be partitioned into two classes: $\mathcal{C}_{\mathrm{w}}=$ $\left\{\sigma_{i} \geq \delta\right\}$ and $\mathcal{C}_{\mathrm{t}}=\left\{\sigma_{i}<\delta\right\}$. The class means of $\mathcal{C}_{\mathrm{w}}$ and $\mathcal{C}_{\mathrm{t}}$ are, respectively,

$$
\begin{aligned}
\mu_{\mathrm{w}}(\delta) & =\frac{1}{\mathcal{P}_{\mathrm{w}}(\delta)} \sum_{i=i_{\delta}}^{L-1} \xi_{i} \mathcal{P}\left(\xi_{i}\right) \\
\mu_{\mathrm{t}}(\delta) & =\frac{1}{\mathcal{P}_{\mathrm{t}}(\delta)} \sum_{i=0}^{i_{\delta}-1} \xi_{i} \mathcal{P}\left(\xi_{i}\right)
\end{aligned}
$$

where $i_{\delta}$ denotes the index of the left endpoint of the interval that includes $\delta$, and $\mathcal{P}_{\mathrm{w}}(\delta)$ and $\mathcal{P}_{\mathrm{t}}(\delta)$ are normalizing constants given by

$$
\mathcal{P}_{\mathrm{w}}(\delta)=\sum_{i=i_{\delta}}^{L-1} \mathcal{P}\left(\xi_{i}\right) \quad \text { and } \quad \mathcal{P}_{\mathrm{w}}(\delta)=\sum_{i=0}^{i_{\delta}-1} \mathcal{P}\left(\xi_{i}\right) .
$$

The total mean of the classes, which is independent of $\delta$, is

$$
\mu_{0}=\sum_{i=0}^{L-1} \xi_{i} \mathcal{P}\left(\xi_{i}\right)
$$

The optimum Otsu threshold is obtained by maximizing the between class variance

$$
\widehat{\delta}=\arg \max _{\delta}\left\{\Sigma_{0}(\delta)\right\},
$$

where the between class variance is given by

$$
\Sigma_{0}(\delta)=\mathcal{P}_{\mathrm{w}}\left[\mu_{\mathrm{w}}(\delta)-\mu_{0}\right]^{2}+\mathcal{P}_{\mathrm{t}}\left[\mu_{\mathrm{t}}(\delta)-\mu_{0}\right]^{2} .
$$

\section{ACKNOWLEDGMENT}

The authors would like to thank Dr. Fauzia Ahmad from the Center of Advanced Communications at Villanova University, Villanova, PA, USA, for providing the experimental data.

\section{REFERENCES}

[1] M. G. Amin, (Ed.), Through-the-wall radar imaging, Boca Raton, FL:CRC Press, 2010.

[2] M. Amin and K. Sarabandi, "Special issue on remote sensing of building interior," IEEE Transactions on Geoscience and Remote Sensing, vol. 47, no. 5, pp. 1267-1268, 2009.

[3] M. Amin, "Special issue on advances in indoor radar imaging," Journal of the Franklin Institute, vol. 345, no. 6, pp. 556-722, 2008.

[4] E. J. Baranoski, "Through-wall imaging: historical perspective and future directions," Journal of the Franklin Institute, vol. 345, no. 6, pp. 556-569, 2008.

[5] M. G. Amin and F. Ahmad, "Wideband synthetic aperture beamforming for through-the-wall imaging," IEEE Signal Processing Magazine, vol. 25, no. 4, pp. 110-113, 2008.

[6] R. Dilsavor, W. Ailes, P. Rush, F. Ahmad, W. Keichel, G. Titi, and M. Amin, "Experiments on wideband through the wall imaging," in Proc. of the SPIE Vol. 5808, Symposium on Defense and Security, Algorithms for Synthetic Aperture Radar Imagery XII Conference, 2005, pp. 196-209.

[7] J. Moulton, S. A. Kassam, F. Ahmad, M. G. Amin, and K. Yemelyanov, "Target and change detection in synthetic aperture radar sensing of urban structures," in Proc. of the IEEE Radar Conference, 2008, pp. 1-6.

[8] F. Ahmad, Y. Zhang, and M. G. Amin, "Three-dimensional wideband beamforming for imaging through a single wall," IEEE Geoscience and Remote Sensing Letters, vol. 5, no. 2, pp. 176-179, 2008.

[9] Y.-S. Yoon and M. G. Amin, "Spatial filtering for wall-clutter mitigation in through-the-wall radar imaging," IEEE Transactions on Geoscience and Remote Sensing, vol. 47, no. 9, pp. 3192-3208, 2009.

[10] M. Dehmollaian and K. Sarabandi, "Analytical, numerical, and experimental methods for through-the-wall radar imaging," in Proc. of the IEEE International Conference on Acoustics, Speech and Signal Processing, 2008, pp. 5181-5184.

[11] M. Dehmollaian and K. Sarabandi, "Refocusing through building walls using synthetic aperture radar," IEEE Transactions on Geoscience and Remote Sensing, vol. 46, no. 6, pp. 1589-1599, 2008.

[12] A. N. Gaikwad, D. Singh, and M. J. Nigam, "Study of effect of room window on through wall imaging in UWB range," in Proc. of the International Conference on Emerging Trends in Electronic and Photonic Devices and Systems, 2009, pp. 395-398.

[13] R. Chandra, A. N. Gaikwad, D. Singh, and M. J. Nigam, "An approach to remove the clutter and detect the target for ultra-wideband throughwall imaging," Journal of Geophysics and Engineering, vol. 5, no. 4, pp. 412-419, 2008.

[14] P. K. Verma, A. N. Gaikwad, D. Singh, and M. J. Nigam, "Analysis of clutter reduction techniques for through wall imaging in UWB range," Progress in Electromagnetics Research B, vol. 17, pp. 29-48, 2009.

[15] M. M. Riaz and A. Ghafoor, "Through-wall image enhancement based on singular value decomposition," International Journal of Antennas and Propagation, vol. 2012, no. Article ID 961829, pp. 1-20, 2012; doi:10.1155/2012/961829

[16] P. Setlur, M. G. Amin, and F. Ahmad, "Analysis of micro-Doppler signals using linear FM basis decomposition," in Proc. of SPIE Vol. 6210, Radar Sensor Technology X, 2006, pp. 62100M 1-11. 
[17] Y. Kim, and H. Ling, "Human activity classification based on microDoppler signatures using a support vector machine," IEEE Transactions on Geoscience Remote Sensing, vol. 47, no. 5, pp. 1328-1337, 2009.

[18] F. Ahmad, M. G. Amin, and P. Setlur, "Through-the-wall target localization using dual-frequency CW radars," in Proc. of SPIE Vol. 6201, Sensors, and Command, Control, Communications, and Intelligence (C3I) Technologies for Homeland Security and Homeland Defense V, 2006, pp. $62010 \mathrm{H} 1-12$.

[19] F. Abujarad, A. Jostingmeier, and A. S. Omar, "Clutter removal for landmine using different signal processing techniques," in Proc. of the Tenth International Conference on Ground Penetrating Radar, 2004, pp. 697-700.

[20] F. Abujarad, G. Nadimy, and A. Omar, "Clutter reduction and detection of landmine objects in ground penetrating radar data using singular value decomposition (SVD)," in Proc. of the Third International Workshop on Advanced Ground Penetrating Radar, 2005, pp. 37-42.

[21] F. H. C. Tivive, M. G. Amin, and A. Bouzerdoum, "Wall clutter mitigation based on eigen-analysis in through-the-wall radar imaging," in Proc. of the International Conference on Digital Signal Processing, 2011, pp. $1-8$.

[22] F. H. C. Tivive, A. Bouzerdoum, and Moeness G. Amin, "An SVDbased approach for mitigating wall reflections in through-the-wall radar imaging," in Proc. IEEE Radar Conference, 2011, pp. 519-524.

[23] L.-P. Song, C. Yu, and Q. H. Lui, "Through-wall imaging (TWI) by radar: 2-D tomographic results and analysis," IEEE Transactions on Geoscience and Remote Sensing, vol. 43, no. 12, pp. 2793-2798, 2005.

[24] M. Dehmollaian, M. Thiel, and K. Sarabandi, "Through-the-wall imaging using differential SAR," IEEE Transactions on Geoscience and Remote Sensing, vol. 47, no. 5, pp. 1289-1296, 2009.

[25] M. Amin and F. Ahmad, "Compressive Sensing for through the wall radar imaging," Journal of Electronic Imaging, vol. 22, no. 3, Article 030901, pp. 1-21, 2013

[26] S. M. Li and X. F. Liu, "Robust adaptive beamforming imaging approach for stepped-frequency through-the-wall radar," Applied Mechanics and Materials, Mechanical and Electronics Engineering III, vol. 130-134, pp. 45-49, 2011; doi: 10.4028/www.scientific.net/AMM.130-134.

[27] Y.-S. Yoon and M. G. Amin, "High-resolution through-the-wall radar imaging using beamspace music," IEEE Transactions on Antennas and Propagation, vol. 56, no. 6, pp. 1763-1774, 2008.

[28] C. A. Balanis, Advanced Engineering Electromagnetics, New York, NY: John Wiley and Sons, 1989

[29] P. Protiva, J. Mrkvica, and J. Macháč, "Time delay estimation of UWB radar signals backscattered from a wall," Microwave and Optical Technology Letters, vol. 53, no. 6, pp. 1444-1450, 2011.

[30] N. Otsu, "A threshold selection method for gray level histograms," IEEE Transactions on Systems, Man, and Cybernetics, vol. 9, no. 1, pp. 62-66, 1979.

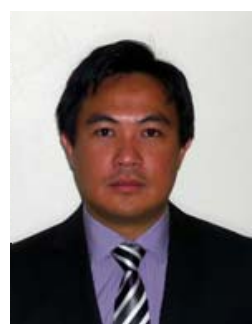

Fok Hing Chi Tivive (M'02) received the Ph.D degree in computer engineering from the University of Wollongong, Australia, in 2006. Since 2006, he has been with the School of Electrical Computer and Telecommunications Engineering, University of Wollongong, as a Postdoctoral Research Fellow. His research interests include machine learning, pattern recognition, image processing, and through-the-wall radar imaging.

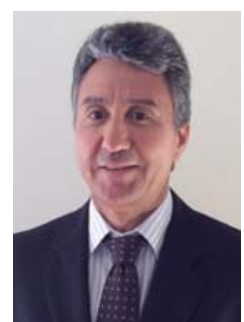

Abdesselam Bouzerdoum (M'89-SM'03) received the M.Sc. and Ph.D. degrees in electrical engineering from the University of Washington, Seattle, USA In 1991 he joined The University of Adelaide, Adelaide, Australia, and in 1998 he was appointed Associate Professor at Edith Cowan University, Perth, Australia. Since 2004, he has been with the University of Wollongong as Professor of Computer Engineering, where he also served as Head of School of Electrical, Computer \& Telecommunications Engineering (2004-2006) and Associate Dean of Research (2007-2013). From 2009 to 2011, he was a Member of the Australian Research Council College of Experts and served as Deputy Chair of the Engineering, Mathematics and Informatics panel from 2010 to 2011. Dr. Bouzerdoum was the recipient of numerous awards and prizes, including the Eureka Prize for Outstanding Science in Support of Defence or National Security in 2011, the Chester Sall Award in 2005, and a Distinguished Researcher Award (Chercheur de Haut Niveau) from the French Ministry of Research in 2001. He has published over 300 technical articles and graduated 34 Ph.D. and Research Masters students. He served as Associate Editor for 4 International journals, including IEEE TRANS. SYSTEMS, MAN, AND CYBERNETICS (1999-2006).

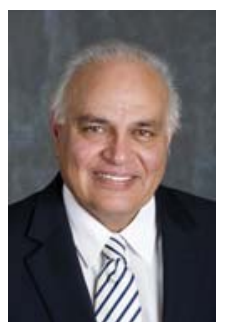

Moeness G. Amin (S'82SM'91M'93F'01) received the $\mathrm{Ph} . \mathrm{D}$. degree in electrical engineering from the University of Colorado, Boulder, CO, USA, in 1984.

Since 1985, he has been a Faculty Member with the Department of Electrical and Computer Engineering, Villanova University, Villanova, PA, USA, where, in 2002, he became the Director of the Center for Advanced Communications, College of Engineering. He has over 600 journal and conference publications in the areas of wireless communications, timefrequency analysis, sensor array processing, waveform design and diversity, interference cancellation in broadband communication platforms, satellite navigations, target localization and tracking, direction finding, channel diversity and equalization, ultrasound imaging, and radar signal processing. He coauthored 18 book chapters. He is the Editor of the book Through the Wall Radar Imaging (CRC Press, 2011) and Compressive Sensing for Urban Radar (CRC Press, 2014). He was a Guest Editor of the Journal of Franklin Institute September 2008 special issue on Advances in Indoor Radar Imaging; Institute of Engineering and Technology (IET) Signal Processing December 2009 special issue on TimeFrequency Approach to Radar Detection, Imaging, and Classification; and the European Association for Signal Processing (EURASIP) Journal on Advances in Signal Processing, special issue on Sparse Sensing in Radar and Sonar Signal Processing in 2014.

Dr. Amin is a Fellow of the International Society of Optical Engineering in 2007 and the IET in 2010. He was a recipient of the Villanova University Outstanding Faculty Research Award in 1997, the IEEE Philadelphia Section Award in 1997, the IEEE ThirdMillennium Medal in 2000, the 2009 Individual Technical Achievement Award from the EURASIP, the 2010 NATO Scientific Achievement Award, and the Chief of Naval Research Challenge Award in 2010. He was a Distinguished Lecturer of the IEEE Signal Processing Society in 20032004 and is currently the Chair of the Electrical Cluster of the Franklin Institute Committee on Science and the Arts. He was a Plenary Speaker at IEEE International Symposium on Signal Processing and Information Technology 2003 (ISSPIT-03), the 2010 IEEE International Conference on Acoustics, Speech and Signal Processing, The 29th International Review of Progress in Applied Computational Electromagnetics 2013 (ACES-13), IET 2013, the 2013 European Signal Processing Conference, Statistics, Optimization, and Signal Processing Workshop 2013 (STATOS-13), IEEE International Workshop on Computational Advances in Multi-Sensor Adaptive Processing 2013 (CAMSAP-13), and International Radar Conference 2014 (RADAR-14). He was a Guest Editor of the IEEE TRANSACTIONS ON GEOSCIENCE AND REMOTE SENSING May 2009 Special Issue on Remote Sensing of Building Interior and the IEEE SIGNAL PROCESSING MAGAZINE November 2013 and July 2014 Special Issues on TimeFrequency Analysis and Applications, and Recent Advances in Synthetic Aperture Radar Imaging. 